JOURNAL OF THE

AMERICAN MATHEMATICAL SOCIETY

Volume 23, Number 1, January 2010, Pages 1-34

S 0894-0347(09)00633-X

Article electronically published on July 6, 2009

\title{
THE SPEED OF PROPAGATION FOR KPP TYPE PROBLEMS. II: GENERAL DOMAINS
}

\author{
HENRI BERESTYCKI, FRANÇOIS HAMEL, AND NIKOLAI NADIRASHVILI
}

\section{Contents}

1. Introduction and main results

1.1. Setting of the problem

1.2. Spreading speeds in general domains

1.3. Dependence on the point $z$

1.4. Dependence on the initial condition

1.5. Comparison with the homogeneous case

1.6. Exterior domains

1.7. Domains containing large half-cylinders

1.8. Further examples

1.9. Other related notions

2. General properties: Dependence on $z$, on $u_{0}$, and general upper bound

2.1. Relationship between $w^{*}\left(e, z, u_{0}\right)$ and $w^{*}\left(e, u_{0}\right)$

2.2. Dependence on the initial datum $\quad 15$

2.3. Upper bound for domains with the extension property 20

3. Exterior domains and domains containing large half-cylinders

4. Domains with zero or infinite spreading speeds, or spreading speeds depending on $z$

4.1. Domains for which $w^{*}\left(e, z, u_{0}\right)$ depends on $z$

4.2. Domains with zero spreading speeds

4.3. Domains with infinite spreading speeds

Acknowledgements

References

\section{Introduction AND MAIN RESUlts}

1.1. Setting of the problem. This paper is concerned with nonlinear spreading and propagation phenomena for reaction-diffusion equations in general unbounded

Received by the editors March 26, 2007.

2000 Mathematics Subject Classification. Primary 35A08, 35B30, 35K05, 35K57; Secondary 35B40, 35K15.

Key words and phrases. Propagation, spreading, reaction-diffusion equations, heat kernel.

Part of this work was carried out during a visit by the first author to the Department of Mathematics of the University of Chicago, the hospitality of which is thankfully acknowledged.

(C)2009 American Mathematical Society Reverts to public domain 28 years from publication 
domains. We consider reaction terms of the Fisher or KPP (for Kolmogorov, Petrovsky, Piskunov) type. Propagation phenomena in a homogeneous framework are well understood, and we will recall below the main results. This article is the second in a series of two, and it is the follow-up of the article [7] (part I). Both papers deal with heterogeneous problems. Part I was concerned with equations with periodic coefficients in domains having periodic structures. The present paper (part II) deals with reaction-diffusion equations with constant coefficients, but in very general domains which are not periodic. We define and analyze various notions of asymptotic spreading speeds for solutions with compactly supported initial data. Before introducing the main notions and stating the main results, let us recall some basic features of the homogeneous framework in $\mathbb{R}^{N}$ and let us also recall some of the results in the periodic framework.

Consider first the Fisher-KPP equation

$$
u_{t}-\Delta u=f(u) \text { in } \mathbb{R}^{N} .
$$

It has been introduced in the celebrated papers of Fisher (1937, [12]) and KPP (1937, [29]) originally motivated by models in biology (in these models, $u$ stands for the concentration of a species). The main assumption is that $f$ is say a $C^{1}\left(\mathbb{R}_{+}\right)$ function satisfying

$$
\left\{\begin{array}{l}
f(0)=f(1)=0, \quad f^{\prime}(1)<0, \quad f^{\prime}(0)>0, \quad f>0 \text { in }(0,1), f<0 \text { in }(1,+\infty), \\
f(s) \leq f^{\prime}(0) s \text { for all } s \in[0,1] .
\end{array}\right.
$$

Archetypes of such nonlinearities are $f(s)=s(1-s)$ or $f(s)=s\left(1-s^{2}\right)$.

Two fundamental features of this equation account for its success in representing propagation (or invasion) and spreading. First, this equation has a family of planar travelling fronts. These are solutions of the form $u(t, x)=U(x \cdot e-c t)$, where $e$ is a fixed vector of unit norm which is the direction of propagation and $c>0$ is the speed of the front. Here $U: \mathbb{R} \mapsto \mathbb{R}$ is given by

$$
-U^{\prime \prime}-c U^{\prime}=f(U) \text { in } \mathbb{R}, \quad U(-\infty)=1, U(+\infty)=0 .
$$

In the original paper of Kolmogorov, Petrovsky and Piskunov, it was proved that, under the above assumptions, there is a threshold value $c^{*}=2 \sqrt{f^{\prime}(0)}>0$ for the speed $c$. Namely, no fronts exist for $c<c^{*}$, and, for each $c \geq c^{*}$, there is a unique front $U$ of the previous type. Uniqueness is up to shift in space or time variables.

Another fundamental property of this equation was established mathematically by Aronson and Weinberger (1978, [1). It deals with the asymptotic speed of spreading. Namely, if $u_{0}$ is a nonnegative continuous function in $\mathbb{R}^{N}$ with compact support and $u_{0} \not \equiv 0$, then the solution $u(t, x)$ of (1.1) with initial condition $u_{0}$ at time $t=0$ spreads with the speed $c^{*}$ in all directions for large times: as $t \rightarrow+\infty$, $\max _{|x| \leq c t}|u(t, x)-1| \rightarrow 0$ for each $c \in\left[0, c^{*}\right)$ and $\max _{|x| \geq c t} u(t, x) \rightarrow 0$ for each $c>c^{*}$.

In Part I of [7] and in an earlier paper [4, we introduced a general heterogeneous periodic framework extending (1.1). The types of equations which were considered there were

$$
u_{t}-\nabla \cdot(A(x) \nabla u)+q(x) \cdot \nabla u=f(x, u) \text { in } \Omega, \quad \nu \cdot A \nabla u=0 \text { on } \partial \Omega,
$$

where $\nu$ denotes the outward unit normal on $\partial \Omega$. Both the coefficients of the equation, namely the diffusion matrix $A(x)$, the drift $q(x)$ and the reaction term $f(x, s)$, as well as the geometry of the underlying connected open set $\Omega$, were 
assumed to be periodic. More precisely, there are $d \in\{1, \ldots, N\}$ and $d$ positive real numbers $L_{1}, \ldots, L_{d}$ such that

$$
\left\{\begin{array}{l}
\forall k \in L_{1} \mathbb{Z} \times \cdots \times L_{d} \mathbb{Z} \times\{0\}^{N-d}, \quad \Omega+k=\Omega \\
\exists C \geq 0, \forall x=\left(x_{i}\right)_{1 \leq i \leq N} \in \bar{\Omega}, \quad\left|x_{d+1}\right|+\cdots+\left|x_{N}\right| \leq C,
\end{array}\right.
$$

and the functions $A, q$ and $f$ are periodic with periods $L_{1}, \ldots, L_{d}$ in the variables $x_{1}, \ldots, x_{d}$. Given a unit direction $e \in \mathbb{R}^{d} \times\{0\}^{N-d}$, a pulsating travelling front in the direction $e$ is a solution $u(t, x)$ of the type $u(t, x)=U(x \cdot e-c t, x)$, where $U=U(s, x)$ is periodic in the variables $x_{1}, \ldots, x_{d}$ (with periods $L_{1}, \ldots, L_{d}$ ) and $U(s, x) \rightarrow 1$ as $s \rightarrow-\infty, U(s, x) \rightarrow 0$ as $s \rightarrow+\infty$, uniformly with respect to $x \in \bar{\Omega}$ (assuming that $f(x, 0)=f(x, 1)=0$ ). Under some natural assumptions on $f$ (generalizing the hypothesis (1.2) ) and on $A$ and $q$, existence and uniqueness (for each speed) of pulsating fronts for, and only for, speeds $c \geq c^{*}(e)$ were proved in [4, 7, 20, 21. A variational formula for the minimal speed $c^{*}(e)$, in terms of some periodic eigenvalue problems, was also derived in 7 . These results extended some earlier results in dimension 1 (see e.g. 25, 38) and in straight infinite cylinders with shear flows [8]. Let us mention here that other types of nonlinearities (combustion type, bistable type, other nonlinearities arising in population dynamics,...) were also dealt with in the literature (see [4, 11, 19, 24, 35, 39, 41, and the references therein for some existence, uniqueness and stability results on fronts in homogeneous or periodic media and formulæ for the speeds of propagation).

Furthermore, the same type of spreading properties holds in the periodic framework as in the homogeneous one. Namely, for problem (1.3) under the assumption that $0<f(x, s) \leq f_{s}^{\prime}(x, 0) s$ for all $s \in(0,1)$ and $x \in \bar{\Omega}$, Freidlin and Gärtner [14] and Freidlin [13] in the case of $\mathbb{R}^{N}$, and then Weinberger [40] in the general periodic framework described above, proved the existence of an asymptotic spreading speed (or ray speed) $w^{*}(e)>0$ such that if $u(t, x)$ solves (1.3) with a nonnegative, continuous and compactly supported initial condition $u_{0} \not \equiv 0$, then

$(1.5)$

$\left\{\begin{aligned} \max _{x \in K, 0 \leq s \leq c t, x+s e \in \bar{\Omega}}|u(t, x+s e)-1| & \rightarrow 0 \text { if } 0 \leq c<w^{*}(e), \\ \max _{x \in K, s \geq c t, x+s e \in \bar{\Omega}} u(t, x+s e) & \rightarrow 0 \text { if } c>w^{*}(e),\end{aligned} \quad\right.$ as $t \rightarrow+\infty$,

for any large enough compact set $K$ so that the sets in which the maxima are taken are not empty. Moreover, $w^{*}(e)$ is given in terms of the minimal speeds of pulsating fronts by the geometrical formula $w^{*}(e)=\min _{\xi \in \mathbb{R}^{d} \times\{0\}^{N-d}, \xi \cdot e>0} c^{*}(\xi) /(e \cdot \xi)$. (See [40; see also [1, 11, 26, 27] for other results with other types of nonlinearities in the homogeneous case, and [34, 36] for equations with shear flows in straight infinite cylinders. Other results, including some with more general time-space scalings, were also obtained in 33. .) The dependence of $c^{*}(e)$ and $w^{*}(e)$ on the coefficients of (1.3) (monotonicity, bounds, asymptotics) is analyzed in Part I of [7] (see also 2, 3, 6, 9, 22, 23, 28, 37, 42,).

We also studied in 7 the influence of the geometry of the periodic domain $\Omega$ (under assumption (1.4)) on the propagation speeds, for the equation

$$
u_{t}=\Delta u+f(u) \text { in } \Omega, \quad \nu \cdot \nabla u=0 \text { on } \partial \Omega
$$

under assumption (1.2) for $f$. More precisely, one of the results was that

$$
w^{*}(e) \leq c^{*}(e) \leq 2 \sqrt{f^{\prime}(0)}
$$


and $w^{*}(e)=2 \sqrt{f^{\prime}(0)}$ if and only if $\Omega$ is invariant in the direction $e$ (straight cylinder in the direction $e$, with bounded or unbounded section). Notice that this geometrical condition is also necessary for the equality $c^{*}(e)=2 \sqrt{f^{\prime}(0)}$ to hold (see [7]). In other words, the presence of holes or of an undulating boundary always hinder the progression or the spreading. Moreover, we proved in [7 that the speeds $c^{*}(e)$ are not in general monotone with respect to the size of the perforations. The inequality $w^{*}(e) \leq c^{*}(e)$ always works. The equality $w^{*}(e)=c^{*}(e)\left(=2 \sqrt{f^{\prime}(0)}\right)$ holds in the homogeneous framework (1.1) in $\mathbb{R}^{N}$, but the inequality $w^{*}(e) \leq c^{*}(e)$ may be strict in general (see Remark 1.12 in [7]).

1.2. Spreading speeds in general domains. Let us now come back to the general nonperiodic case and deal with the Cauchy problem for the Fisher-KPP equation:

$$
\left\{\begin{aligned}
u_{t} & =\Delta u+f(u) & & \text { in } \Omega, t>0, \\
\nu \cdot \nabla u & =0 & & \text { on } \partial \Omega, t>0, \\
u(0, x) & =u_{0}(x) & & \text { in } \Omega .
\end{aligned}\right.
$$

Throughout the paper, $\Omega$ denotes a domain (open connected subset) of $\mathbb{R}^{N}$, locally $C^{2}$, with outward unit normal $\nu$. The initial condition $u_{0}$ is continuous, nonnegative, $u_{0} \not \equiv 0$ in $\bar{\Omega}$ and $u_{0}$ is compactly supported in $\bar{\Omega}$. One calls $\mathcal{E}$ the set of such functions $u_{0}$. The $C^{1}$ function $f: \mathbb{R}_{+} \rightarrow \mathbb{R}$ is assumed to satisfy (1.2). This assumption on $f$ is made from now on throughout the paper. The function $u(t, x)$ is defined as the nondecreasing limit, as $n \rightarrow+\infty$, of the functions $u^{n}(t, x)$ which are weak solutions of the equation $u_{t}^{n}=\Delta u^{n}+f\left(u^{n}\right)$ in $\Omega \cap B_{n}$ for $t>0$, with boundary condition $\nu \cdot \nabla u^{n}=0$ on $\partial \Omega \cap B_{n}, u^{n}=0$ on $\bar{\Omega} \cap \partial B_{n}$ and initial condition $u^{n}(0, \cdot)=u_{0} \bar{\Omega} \cap \overline{B_{n}}$. Here, $B_{r}$ denotes the open euclidean ball of $\mathbb{R}^{N}$ with centre 0 and radius $r>0$. Notice that $u$ is a classical solution of (1.6) for all $t>0$ and that $0<u(t, x)<\max \left(\max _{\bar{\Omega}} u_{0}, 1\right)$, for all $t>0$ and $x \in \bar{\Omega}$, from the maximum principle.

Traveling or pulsating fronts do not exist anymore in this general nonperiodic framework, even if the notion of fronts can be extended to arbitrary geometries (see [5]). But the purpose of this paper is rather, first, to understand how we can extend the notions of asymptotic spreading speeds for the solutions of the Cauchy problem (1.6) with a compactly supported initial condition $u_{0} \in \mathcal{E}$. Different definitions can be given, which are coherent with the periodic case. We then analyze the relationships between these general new definitions. Some other fundamental questions will then be asked: how do the spreading speeds depend on the initial condition? Can they be compared to the spreading speed $2 \sqrt{f^{\prime}(0)}$ of the whole space $\mathbb{R}^{N}$ ? We will especially see that the answer to this last question is positive for a large class of domains, but is negative in some domains for which the spreading speed is infinite. We also analyze in detail the case of exterior domains.

Let us now make more precise the definitions of spreading speeds in unbounded directions of $\Omega$. In all that follows, one calls $B(z, r)$ the open euclidean ball of centre $z$ and radius $r$ in $\mathbb{R}^{N}$. In the following, we also take the convention that, for a function $v: E \subset \mathbb{R}^{m} \rightarrow \mathbb{R}, \sup _{\emptyset} v=+\infty$.

Definition 1.1. We say that a connected open set $\Omega \subset \mathbb{R}^{N}$ is strongly unbounded in a direction $e \in \mathbb{S}^{N-1}$ if there exist $R_{0} \geq 0$ and $s_{0} \in \mathbb{R}$ such that $\overline{B\left(s e, R_{0}\right)} \cap \bar{\Omega} \neq \emptyset$ for all $s \geq s_{0}$. With a slight abuse of notation, we set $\overline{B(y, 0)}=\{y\}$ for all $y \in \mathbb{R}^{N}$. 
We then define $R(e) \geq 0$ as

$$
R(e)=\inf \left\{R \geq 0, \exists s \in \mathbb{R}, \forall s^{\prime} \geq s, \quad \overline{B\left(s^{\prime} e, R\right)} \cap \bar{\Omega} \neq \emptyset\right\} .
$$

As an example, a periodic domain $\Omega$, satisfying (1.4), is strongly unbounded in any unit direction $e \in \mathbb{R}^{d} \times\{0\}^{N-d}$.

Since problem (1.6) is well-understood when $N=1$ (in which case strong unboundedness in the direction \pm 1 means that $\Omega \supset \pm[a,+\infty)$ for some $a \in \mathbb{R}$, since $\Omega$ is always assumed to be connected), one can assume that $N \geq 2$ in the sequel.

Definition 1.2. Let $e$ be a direction in which $\Omega$ is strongly unbounded and let $R(e) \geq 0$ be as in Definition 1.1. Let $u$ be the solution of (1.6) with initial condition $u_{0} \in \mathcal{E}$.

We define the spreading speed of $u$ in the direction $e$ as

$$
w^{*}\left(e, u_{0}\right)=\inf \left\{c>0, \forall A>R(e), \limsup _{t \rightarrow+\infty}\left[\sup _{s \geq c t, x \in \overline{B(s e, A)} \cap \bar{\Omega}} u(t, x)\right]=0\right\} .
$$

We set $w^{*}\left(e, u_{0}\right)=+\infty$ if there is no $c>0$ such that $\sup _{s \geq c t, x \in \overline{B(s e, A)} \cap \bar{\Omega}} u(t, x) \rightarrow$ 0 as $t \rightarrow+\infty$ for all $A>R(e)$.

The nonnegative real number $w^{*}\left(e, u_{0}\right)$, if finite, can be viewed as the asymptotic speed of the leading edge of the solution $u$ uniformly with respect to all cylinders along the direction $e$.

Another related notion, which is more precise in some sense, is that of spreading speed along a half-line.

Definition 1.3. Under the same assumptions as in Definition 1.2 we define the spreading speed of $u$ along the half-line $z+\mathbb{R}_{+} e$, for $z \in \mathbb{R}^{N}$, as

$$
w^{*}\left(e, z, u_{0}\right)=\inf \left\{c>0, \exists A>0, \limsup _{t \rightarrow+\infty}\left[\sup _{s \geq c t, x \in \overline{B(z+s e, A)} \cap \bar{\Omega}} u(t, x)\right]=0\right\} .
$$

We set $w^{*}\left(e, z, u_{0}\right)=+\infty$ if for all $c>0$ and $A>0, \sup _{s \geq c t, x \in \overline{B(z+s e, A)} \cap \bar{\Omega}} u(t, x)$ $\nrightarrow \rightarrow 0$ as $t \rightarrow+\infty$.

The nonnegative real number $w^{*}\left(e, z, u_{0}\right)$, if finite, is the asymptotic spreading speed of $u$ locally along the line $z+\mathbb{R}_{+} e$. Notice also that $w^{*}\left(e, z, u_{0}\right)=$ $w^{*}\left(e, z+\sigma e, u_{0}\right)$ for all $\sigma \in \mathbb{R}$.

Remark 1.4. Under the above notations, say

$$
R(e, z)=\inf \left\{R \geq 0, \exists s \in \mathbb{R}, \forall s^{\prime} \geq s, \quad \overline{B\left(z+s^{\prime} e, R\right)} \cap \bar{\Omega} \neq \emptyset\right\} .
$$

Notice that $R(e, 0)=R(e)$ and that $R(e)-|z-(z \cdot e) e| \leq R(e, z) \leq R(e)+|z-(z \cdot e) e|$ for all $z \in \mathbb{R}^{N}$. If $R(e, z)>0$ and if there exists $s \in \mathbb{R}$ such that $\overline{B\left(z+s^{\prime} e, R(e, z)\right)} \cap$ $\bar{\Omega} \neq \emptyset$ for all $s^{\prime} \geq s \mathbb{1}$ then the definition of $w^{*}\left(e, z, u_{0}\right)$ is equivalent to the following one:

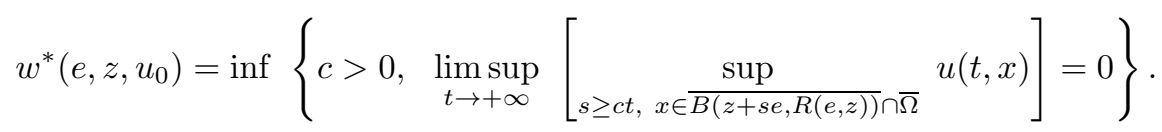

\footnotetext{
${ }^{1}$ Notice that the existence of such a real number $s$ is not guaranteed in general, as the following example shows: in $\mathbb{R}^{2}$, call $x_{k}=\left(k^{2}, 0\right)$ for $k \in \mathbb{N}$ and set $\Omega=\mathbb{R}^{2} \backslash \bigcup_{k \in \mathbb{N}} B\left(x_{k}, 1+1 / k\right)$. For $e=$ $(1,0)$ and $z=(0,0)$ one has $R(e)=R(e, z)=1$, but there is no $s \in \mathbb{R}$ such that $\overline{B\left(z+s^{\prime} e, 1\right)} \cap \bar{\Omega} \neq$ $\emptyset$ for all $s^{\prime} \geq s$.
} 
In the case where $R(e, z)=0$ or if there is no $s \in \mathbb{R}$ such that $\overline{B\left(z+s^{\prime} e, R(e, z)\right)} \cap$ $\bar{\Omega} \neq \emptyset$ for all $s^{\prime} \geq s$, then the definition of $w^{*}\left(e, z, u_{0}\right)$ is equivalent to the following one:

$$
w^{*}\left(e, z, u_{0}\right)=\inf \left\{c>0, \exists A>R(e, z), \limsup _{t \rightarrow+\infty}\left[\sup _{s \geq c t, x \in \overline{B(z+s e, A)} \cap \bar{\Omega}} u(t, x)\right]=0\right\} .
$$

Furthermore, it immediately follows from the above definitions that

$$
\forall \gamma>w^{*}\left(e, u_{0}\right), \forall A>R(e), \quad \max _{x \in \overline{B(\gamma t e, A)} \cap \bar{\Omega}} u(t, x) \rightarrow 0 \text { as } t \rightarrow+\infty
$$

and that

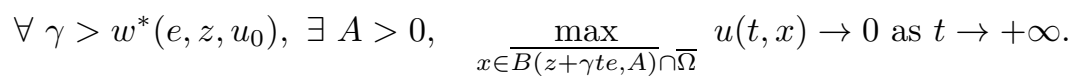

If $\Omega$ is a periodic domain satisfying (1.4), then these new notions of asymptotic spreading speeds are coherent with the previous one, $w^{*}(e)$, characterized by (1.5); namely,

$$
w^{*}\left(e, z, u_{0}\right)=w^{*}\left(e, u_{0}\right)=w^{*}(e)
$$

for all $u_{0} \in \mathcal{E}$, for all $z \in \mathbb{R}^{N}$ and for all unit direction $e \in \mathbb{R}^{d} \times\{0\}^{N-d}$.

1.3. Dependence on the point $z$. In general nonperiodic domains, it is clear that the inequality

$$
w^{*}\left(e, z, u_{0}\right) \leq w^{*}\left(e, u_{0}\right)
$$

holds for all $z \in \mathbb{R}^{N}$. However, the inequality may be strict, as the following proposition shows. We can furthermore make more precise the relationship between $w^{*}\left(e, u_{0}\right)$ and $w^{*}\left(e, z, u_{0}\right)$ when $z$ varies.

Proposition 1.5. Let $N \geq 2$ and $e \in \mathbb{S}^{N-1}$ be given. For each locally $C^{2}$ domain $\Omega$ which is strongly unbounded in the direction $e$ and for each initial condition $u_{0} \in \mathcal{E}$, one has

$$
\sup _{z \in \mathbb{R}^{N}} w^{*}\left(e, z, u_{0}\right)=w^{*}\left(e, u_{0}\right) .
$$

Furthermore, given $z \in \mathbb{R}^{N}$, there are some locally $C^{2}$ domains $\Omega$ which are strongly unbounded in the direction $e$ and such that $w^{*}\left(e, z, u_{0}\right)<w^{*}\left(e, u_{0}\right)$ for all $u_{0} \in \mathcal{E}$.

The proof of the second assertion relies on some precise heat kernel estimates as well as on some lower bounds of $w^{*}\left(e, u_{0}\right)$ for some domains containing a "quarter of spaces" (see Proposition 1.11 and Corollary 1.12 below). We actually prove more than what is stated here: namely, up to translation and rotation, we exhibit some domains $\Omega$ for which $w^{*}\left(e, u_{0}\right)=2 \sqrt{f^{\prime}(0)}$ for all $u_{0} \in \mathcal{E}$ and $w^{*}\left(e, z, u_{0}\right)=0$ for all $u_{0} \in \mathcal{E}$ and for all $z \in \mathbb{R}^{N}$, such that $z \cdot e^{\prime}>h$ (here, $e^{\prime} \in \mathbb{S}^{N-1}$ is any given direction which is orthogonal to $e$, and $h$ is any given real number).

In the following result, we give a sufficient condition for the spreading speed $w^{*}\left(e, z, u_{0}\right)$ not to depend on $z$. Let us first introduce the notation $H_{y, z}$ and the definition of global $C^{2, \alpha}$ smoothness of $\Omega$ :

Hypothesis $H_{y, z}$. Let $\Omega$ be strongly unbounded in a direction $e \in \mathbb{S}^{N-1}$. We say that $y$ and $z \in \mathbb{R}^{N}$ are asymptotically connected in the direction $e$ within $\Omega$ (or 
that Hypothesis $H_{y, z}$ is satisfied) if there exist $R_{y}>R(e, y)$ and $R_{z}>R(e, z)$ such that

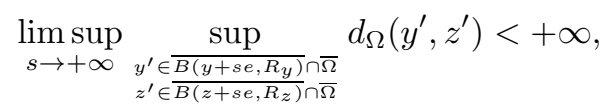

where $d_{\Omega}$ denotes the geodesic distance in $\bar{\Omega}$. This condition is a type of relative connectedness assumption in the direction $e$. A typical counter-example is what we call Luckhaus's comb 2 Notice that Hypothesis $H_{y, z}$ is satisfied for any $y, z \in \mathbb{R}^{N}$ if $\Omega$ is a periodic domain of the type (1.4). Lastly, if Hypothesis $H_{y, z}$ is satisfied, then so is $H_{y+\alpha e, z+\beta e}$ for any $(\alpha, \beta) \in \mathbb{R}^{2}$ (see Remark 2.1 for the details).

Global $C^{2, \alpha}$ smoothness of $\Omega$. Call $B_{s}^{N-1}$ the closed Euclidean ball of $\mathbb{R}^{N-1}$ with center 0 and radius $s>0$. In the entire paper, if $\Omega \subset \mathbb{R}^{N}$ is a nonempty connected open subset of $\mathbb{R}^{N}$ and if $\alpha$ is a positive real number, we say that $\Omega$ is globally of class $C^{2, \alpha}$ if there exist $r>0$ and $M>0$ such that, for all $z \in \partial \Omega$, there is a linear isometric map $T_{z}: \mathbb{R}^{N} \rightarrow \mathbb{R}^{N}$ and there is a $C^{2, \alpha}$ map $\phi_{z}: B_{2 r}^{N-1} \rightarrow \mathbb{R}$ such that $\phi_{z}(0)=0,\left\|\phi_{z}\right\|_{C^{2, \alpha}\left(B_{2 r}^{N-1}\right)} \leq M$ and

$$
\begin{gathered}
\Omega \cap B(z, r)=\left[z+T_{z}\left(\left\{y \in \mathbb{R}^{N},\left(y_{1}, \ldots, y_{N-1}\right) \in B_{2 r}^{N-1},\right.\right.\right. \\
\left.\left.\left.\phi_{z}\left(y_{1}, \ldots, y_{N-1}\right)<y_{N}\right\}\right)\right] \cap B(z, r) .
\end{gathered}
$$

Notice in particular that $\mathbb{R}^{N}$ is globally of class $C^{2, \alpha}$ for all $\alpha>0$.

Theorem 1.6 (Dependence on $z$ ). Let $N \geq 2$ and $e \in \mathbb{S}^{N-1}$ be given. Assume that $\Omega$ is strongly unbounded in the direction e, that $\Omega$ is globally of class $C^{2, \alpha}$ for some $\alpha>0$, and that Hypothesis $H_{y, z}$ is satisfied for some $y$ and $z$ in $\mathbb{R}^{N}$. Then

$$
\forall u_{0} \in \mathcal{E}, \quad w^{*}\left(e, y, u_{0}\right)=w^{*}\left(e, z, u_{0}\right) .
$$

As a consequence, if $\Omega$ satisfies Hypothesis $H_{y, z}$ for all points $y$ and $z$ in $\mathbb{R}^{N}$, then $w^{*}\left(e, z, u_{0}\right)=w^{*}\left(e, u_{0}\right)$ for all $z \in \mathbb{R}^{N}$ and $u_{0} \in \mathcal{E}$.

1.4. Dependence on the initial condition. Some other fundamental questions concern the possible a priori dependence of $w^{*}\left(e, u_{0}\right)$ or $w^{*}\left(e, z, u_{0}\right)$ on the initial condition $u_{0} \in \mathcal{E}$, as well as some bounds so for the spreading speeds. For periodic domains satisfying (1.4), one recalls that the spreading speeds do not depend on $u_{0}$ (or on $z$ ) and are bounded from above by $2 \sqrt{f^{\prime}(0)}$.

The following theorem provides a general sufficient condition on $\Omega$ so that the spreading speeds $w^{*}\left(e, u_{0}\right)$ and $w^{*}\left(e, z, u_{0}\right)$ not to depend on $u_{0}$.

Theorem 1.7 (Dependence on $u_{0}$ ). Let $\Omega$ be a connected open subset of $\mathbb{R}^{N}$ and assume that $\Omega$ is globally of class $C^{2, \alpha}$ for some $\alpha>0$. Assume also that for all $r \geq 0$

$$
\sup _{(x, y) \in \bar{\Omega}^{2},|x-y| \leq r} d_{\Omega}(x, y)<+\infty .
$$

Let $\mu_{r}^{z}$ denote the Lebesgue measure of $\Omega \cap B(z, r)$ for each $z \in \mathbb{R}^{N}$ and $r>0$. Assume that there exists $R_{0}>0$ such that $\mu_{r}^{z}>0$ for all $z \in \mathbb{R}^{N}$ and $r \geq R_{0}$, and that $\mu_{r+1}^{z} / \mu_{r}^{z} \rightarrow 1$ as $r \rightarrow+\infty$, uniformly in $z \in \mathbb{R}^{N}$.

Let $u$ be the solution of (1.6) with a given initial condition $u_{0} \in \mathcal{E}$. Then $u(t, x) \rightarrow$ 1 uniformly locally in $x \in \bar{\Omega}$ as $t \rightarrow+\infty$. Furthermore, $\Omega$ is strongly unbounded in

\footnotetext{
${ }^{2}$ Luckhaus's comb is the example we use in the second part of Proposition 1.5 above (see Section 4.1 for the definition).
} 
any direction $e \in \mathbb{S}^{N-1}$ and $w^{*}\left(e, u_{0}\right)$ and $w^{*}\left(e, z, u_{0}\right)$ do not depend on the initial condition $u_{0}$, provided that $u_{0}<1$.

Note that the hypothesis (1.8) is a type of strong connectedness assumption for the domain $\Omega$. Once again, a typical counterexample is Luckhaus's comb (see Section 4.1).

1.5. Comparison with the homogeneous case. As far as bounds for the spreading speeds are concerned, the speed $2 \sqrt{f^{\prime}(0)}$, which is the spreading speed if $\Omega=\mathbb{R}^{N}$, bounds from above the spreading speed if $\Omega$ is a periodic domain satisfying (1.4). Furthermore, we prove here that the same property turns out to be true for the large class of domains satisfying the extension property. This class of domain is defined now: quoting Davies [10, a nonempty open subset $\Omega$ of $\mathbb{R}^{N}$ is said to have the extension property if, for all $1 \leq p \leq+\infty$, there exists a bounded linear map $E$ from $W^{1, p}(\Omega)$ into $W^{1, p}\left(\mathbb{R}^{N}\right)$ such that $E(f)$ is an extension of $f$ from $\Omega$ to $\mathbb{R}^{N}$ for all $f \in W^{1, p}(\Omega)$. This property is equivalent to the existence of $\varepsilon>0, k \in \mathbb{N}, M>0$ and of a countable sequence of open sets $\left(U_{n}\right)_{n \in \mathbb{N}}$ such that:

(i) if $x \in \partial \Omega$, then the ball with centre $x$ and radius $\varepsilon$ is contained in $U_{n}$ for some $n$,

(ii) no point in $\mathbb{R}^{N}$ is contained in more than $k$ distinct sets $U_{n}$,

(iii) for each $n$, there exists an isometry $T_{n}: \mathbb{R}^{N} \rightarrow \mathbb{R}^{N}$ and a Lipschitzcontinuous function $\phi_{n}: \mathbb{R}^{N-1} \rightarrow \mathbb{R}$ whose Lipschitz norm is bounded by $M$. Moreover, $U_{n} \cap \Omega=U_{n} \cap T_{n} \Omega_{n}$, where

$$
\Omega_{n}=\left\{\left(z_{1}, \ldots, z_{N}\right) \in \mathbb{R}^{N}, \phi_{n}\left(z_{1}, \ldots, z_{N-1}\right)<z_{N}\right\} .
$$

Any smooth bounded or exterior domain satisfies the extension property. So does any smooth periodic domain.

Theorem 1.8 (General upper bound). Let $\Omega$ be a locally $C^{2}$ connected open subset of $\mathbb{R}^{N}$ satisfying the extension property. Assume that $\Omega$ is strongly unbounded in a direction $e$. Let $u$ be the solution of (1.6) with a given initial condition $u_{0} \in \mathcal{E}$. Then

$$
w^{*}\left(e, u_{0}\right) \leq 2 \sqrt{f^{\prime}(0)}
$$

and

$$
\forall c>2 \sqrt{f^{\prime}(0)}, \quad \max _{|x| \geq c t, x \in \bar{\Omega}} u(t, x) \rightarrow 0 \text { as } t \rightarrow+\infty .
$$

Under the assumptions of Theorem 1.8, inequality (1.9) yields in particular

$$
w^{*}\left(e, z, u_{0}\right) \leq 2 \sqrt{f^{\prime}(0)}
$$

for all $z \in \mathbb{R}^{N}$. Notice that property (1.10) is actually stronger than (1.9). Theorem 1.8 means that, for the large class of domains satisfying the extension property, the minimal speed of planar fronts, $2 \sqrt{f^{\prime}(0)}$, turns out to be an upper bound for the asymptotic spreading speeds in any direction $e$ in which $\Omega$ is strongly unbounded, as for periodic domains.

Furthermore, as already underlined, for a periodic domain $\Omega$ satisfying (1.4), for any unit vector $e \in \mathbb{R}^{d} \times\{0\}^{N-d}$ and for any $u_{0} \in \mathcal{E}$, inequality (1.9) is an equality if and only if $\Omega$ is a cylinder in direction $e$. However, this property is not true for general domains, as the following subsection shows. 
1.6. Exterior domains. A domain $\Omega \subset \mathbb{R}^{N}$ is called exterior if $\Omega$ is a nonempty connected open subset of $\mathbb{R}^{N}$ such that $\mathbb{R}^{N} \backslash \Omega$ is compact. The simplest example of an exterior domain is the whole space $\mathbb{R}^{N}$.

Theorem 1.9 (Exterior domain). Let $\Omega$ be an exterior domain of class $C^{2}$. Then,

$$
\forall e \in \mathbb{S}^{N-1}, \forall z \in \mathbb{R}^{N}, \forall u_{0} \in \mathcal{E}, \quad w^{*}\left(e, z, u_{0}\right)=w^{*}\left(e, u_{0}\right)=2 \sqrt{f^{\prime}(0)} .
$$

Furthermore, if u solves (1.6) with $u_{0} \in \mathcal{E}$, one has

$$
\left\{\begin{array}{ll}
\forall 0 \leq c<2 \sqrt{f^{\prime}(0)}, & \max _{|x| \leq c t, x \in \bar{\Omega}}|u(t, x)-1| \rightarrow 0, \\
\forall c>2 \sqrt{f^{\prime}(0)}, & \max _{|x| \geq c t, x \in \bar{\Omega}} u(t, x) \rightarrow 0,
\end{array} \quad \text { as } t \rightarrow+\infty .\right.
$$

Remark 1.10. The second property is clearly stronger than the first one. Theorem 1.9 actually extends the classical result of Aronson and Weinberger 1 mentioned above, which was concerned with the case of the whole space $\mathbb{R}^{N}$.

1.7. Domains containing large half-cylinders. The arguments which are used in the proof of Theorem 1.9 imply that if $\Omega$ contains a semi-infinite cylinder in the direction $e$ with a large enough section, then $w^{*}\left(e, u_{0}\right)$ is bounded from below by a constant close to $2 \sqrt{f^{\prime}(0)}$. Here, $\Omega$ always denotes a nonempty connected open subset of $\mathbb{R}^{N}$ of class $C^{2}$ (locally). More precisely, one has the following:

Proposition 1.11 (Lower bounds for domains containing large semi-infinite cylinders). Given $\varepsilon>0$, there exists $R_{0}=R_{0}(\varepsilon)>0$ such that if

$$
\begin{aligned}
\Omega \supset \mathcal{C}_{e, A, x_{0}, R} & :=\left\{x \in \mathbb{R}^{N}, x \cdot e>A,\left|\left(x-x_{0}\right)-\left(\left(x-x_{0}\right) \cdot e\right) e\right|<R\right\} \\
& =\left(\bigcup_{s \in \mathbb{R}} B\left(x_{0}+s e, R\right)\right) \cap\{x \cdot e>A\}
\end{aligned}
$$

for some $e \in \mathbb{S}^{N-1}, A \in \mathbb{R}, x_{0} \in \mathbb{R}^{N}$ and $R>R_{0}$, then

$$
w^{*}\left(e, u_{0}\right) \geq 2 \sqrt{f^{\prime}(0)}-\varepsilon \text { and } w^{*}\left(e, z, u_{0}\right) \geq 2 \sqrt{f^{\prime}(0)}-\varepsilon
$$

for all $u_{0} \in \mathcal{E}$ and $z \in \mathbb{R}^{N}$ such that $\left|z-x_{0}-\left(\left(z-x_{0}\right) \cdot e\right) e\right|<R$.

Thus, if $\Omega$ contains a sequence of semi-infinite cylinders of type $\left(\mathcal{C}_{e, A_{n}, x_{0, n}, R_{n}}\right)_{n \in \mathbb{N}}$ with $A_{n} \in \mathbb{R}, x_{0, n} \in \mathbb{R}^{N}$ and $R_{n} \rightarrow+\infty$ as $n \rightarrow+\infty$, then $w^{*}\left(e, u_{0}\right) \geq 2 \sqrt{f^{\prime}(0)}$ for all $u_{0} \in \mathcal{E}$.

Notice that assumption (1.12) automatically implies that $\Omega$ is strongly unbounded in the direction $e$. Furthermore, the property of containing a sequence of such semi-infinite cylinders holds especially if $\Omega$ contains a "quarter of space":

Corollary 1.12. If $\Omega$ satisfies

$$
\Omega \supset\left\{x \in \mathbb{R}^{N}, x \cdot e>A, x \cdot e^{\prime}>B\right\}
$$

for some $(A, B) \in \mathbb{R}^{2}$ and $e, e^{\prime} \in \mathbb{S}^{N-1}$ with $e^{\prime} \cdot e=0$, then $w^{*}\left(e, u_{0}\right) \geq 2 \sqrt{f^{\prime}(0)}$ and $w^{*}\left(e, z, u_{0}\right) \geq 2 \sqrt{f^{\prime}(0)}$ for all $u_{0} \in \mathcal{E}$ and $z \in \mathbb{R}^{N}$ such that $z \cdot e^{\prime}>B$.

Exterior domains are typical examples of domains satisfying (1.14). Notice from the above corollary and Theorem 1.8 that if $\Omega$ is a locally $C^{2}$ connected open subset of $\mathbb{R}^{N}$ satisfying (1.14) and the extension property, then $w^{*}\left(e, u_{0}\right)=2 \sqrt{f^{\prime}(0)}$ for all $u_{0} \in \mathcal{E}$. 
1.8. Further examples. As already underlined, any periodic domain $\Omega$ satisfying (1.4) is such that $0<w^{*}\left(e, u_{0}\right) \leq 2 \sqrt{f^{\prime}(0)}$ for all unit vector $e \in \mathbb{R}^{d} \times\{0\}^{N-d}$ and for all $u_{0} \in \mathcal{E}$. Furthermore, the upper bound holds for a large class of domains (see Theorem 1.8). However, the following theorem asserts that the spreading speeds $w^{*}\left(e, u_{0}\right)$ and $w^{*}\left(e, z, u_{0}\right)$ may be zero or infinite for some domains $\Omega$. For the sake of clarity, we recall that only open connected sets $\Omega$ are considered.

Theorem 1.13 (Domains with zero or infinite spreading speeds). a) There are some locally $C^{2}$ domains of $\mathbb{R}^{2}$ which satisfy the extension property and are strongly unbounded in every direction $e \in \mathbb{S}^{1}$, and such that $w^{*}\left(e, z, u_{0}\right)=w^{*}\left(e, u_{0}\right)=0$ for all $e \in \mathbb{S}^{1}, z \in \mathbb{R}^{2}$ and $u_{0} \in \mathcal{E}$.

b) For every $N \geq 2$ and $e \in \mathbb{S}^{N-1}$, there are some locally $C^{2}$ domains of $\mathbb{R}^{N}$ which do not satisfy the extension property, and such that $w^{*}\left(e, z, u_{0}\right)=w^{*}\left(e, u_{0}\right)=$ $+\infty$ for all $z \in \mathbb{R}^{N}$ and $u_{0} \in \mathcal{E}$.

Therefore, even in the class of domains satisfying the extension property, there are domains for which the asymptotic speeds $w^{*}\left(e, z, u_{0}\right)$ and $w^{*}\left(e, u_{0}\right)$ are zero in any direction $e$ (such a phenomenon does not happen under the periodicity condition (1.4)). In the proof of Theorem 1.13, we construct domains which have the shape of a spiral and for which the asymptotic spreading speeds are zero in all directions.

Furthermore, there is no universal upper bound without the extension property. Some domains with an infinite cusp have infinite spreading speeds (see the proof of Theorem 1.13 part b). For such domains, we prove some new specific lower bounds for the heat kernel (see Lemma 4.2 in Section 4.3 below).

1.9. Other related notions. Here, we would like to mention some other notions of spreading speeds. We compare them to the notions introduced in Definitions 1.2 and 1.3 and state their main properties.

First, given a nonempty connected locally $C^{2}$ open subset $\Omega$ of $\mathbb{R}^{N}$ and given $e \in \mathbb{S}^{N-1}$ and $u_{0} \in \mathcal{E}$, we can define the asymptotic spreading speed of the leading edge of the solution $u$ of (1.6) in the direction $e$, uniformly with respect to the directions which are orthogonal to $e$, as

$$
w^{* *}\left(e, u_{0}\right)=\inf \left\{c>0, \limsup _{t \rightarrow+\infty} \sup _{x \cdot e \geq c t, x \in \bar{\Omega}} u(t, x)=0\right\},
$$

provided that $\Omega$ satisfies

$$
\exists s \in \mathbb{R}, \forall s^{\prime} \geq s, \quad\left\{x \in \mathbb{R}^{N}, x \cdot e \geq s^{\prime}\right\} \cap \bar{\Omega} \neq \emptyset .
$$

Notice that if $\Omega$ is strongly unbounded in the direction $e$ in the sense of Definition 1.1. then assumption (1.15) is immediately satisfied. This notion of asymptotic spreading speed $w^{* *}\left(e, u_{0}\right)$ is rougher than the previous ones, $w^{*}\left(e, u_{0}\right)$ or $w^{*}\left(e, z, u_{0}\right)$, and it does not give a precise description of where or in which precise direction the leading edge of the solution $u$ moves. However, we can compare it to the previous notions $w^{*}\left(e, u_{0}\right)$ and $w^{*}\left(e, z, u_{0}\right)$, and we can derive some properties of $w^{* *}\left(e, u_{0}\right)$ from the above results.

It is immediate to check that if $\Omega$ satisfies (1.15) and if it is strongly unbounded in a direction $e^{\prime} \in \mathbb{S}^{N-1}$ such that $e^{\prime} \cdot e>0$, then

$\forall u_{0} \in \mathcal{E}, \forall z \in \mathbb{R}^{N}, \quad w^{* *}\left(e, u_{0}\right) \geq w^{*}\left(e^{\prime}, u_{0}\right) \times\left(e^{\prime} \cdot e\right)\left(\geq w^{*}\left(e^{\prime}, z, u_{0}\right) \times\left(e^{\prime} \cdot e\right)\right)$. 
It may then happen that $w^{* *}\left(e, u_{0}\right)>w^{*}\left(e, u_{0}\right)$ for all $u_{0} \in \mathcal{E}$. For instance, in $\mathbb{R}^{2}$, call $H=\left\{x \in \mathbb{R}^{2}, x_{2}-x_{1}>0\right\}$, let $\left(a_{n}\right)_{n \in \mathbb{N}^{*}}$ be a sequence of negative numbers such that $a_{n} / n \rightarrow-\infty$ as $n \rightarrow+\infty$, let

$$
\begin{array}{r}
\Gamma=\bigcup_{n \in \mathbb{N}}\left([2 n, 2 n+1] \times\{0\} \cup[2 n+1,2 n+2] \times\left\{a_{n+1}\right\}\right. \\
\left.\cup\{2 n+1,2 n+2\} \times\left[a_{n+1}, 0\right]\right)
\end{array}
$$

and let $\Omega$ be a globally smooth open connected domain satisfying the extension property and such that

$$
H \cup \Gamma \subset \Omega \subset\left\{x \in \mathbb{R}^{2}, d(x, H \cup \Gamma)<1 / 3\right\},
$$

where $d(x, E)$ denotes the euclidean distance of a point $x$ to a set $E$. With $e=(1,0)$ and $e^{\prime}=(1 / \sqrt{2}, 1 / \sqrt{2})$, one can check that $w^{*}\left(e, u_{0}\right)=0$ for all $u_{0} \in \mathcal{E}$ (by using the same arguments as in the proofs of Theorem 1.8 or Theorem 1.13, part a)), while $w^{*}\left(e^{\prime}, u_{0}\right)=2 \sqrt{f^{\prime}(0)}$ for all $u_{0} \in \mathcal{E}$ (because of Theorem 1.8 and Corollary 1.12). Thus,

$$
\forall u_{0} \in \mathcal{E}, \quad w^{* *}\left(e, u_{0}\right) \geq \sqrt{2} \sqrt{f^{\prime}(0)}>0=w^{*}\left(e, u_{0}\right) .
$$

Furthermore, with the same arguments as in the proofs of Theorems 1.7, 1.8, 1.9 and 1.13, the following properties hold:

1) if $\Omega$ satisfies the general assumptions of Theorem 1.7, then assumption (1.15) is satisfied for all $e \in \mathbb{S}^{N-1}$ and $w^{* *}\left(e, u_{0}\right)$ does not depend on $u_{0} \in \mathcal{E}$, provided that $u_{0}<1$;

2) if $\Omega$ satisfies the assumptions of Theorem 1.8 (extension property), then, because of (1.10), $w^{* *}\left(e, u_{0}\right) \leq 2 \sqrt{f^{\prime}(0)}$ for all $u_{0} \in \mathcal{E}$ and for any direction $e \in$ $\mathbb{S}^{N-1}$ such that (1.15) holds;

3 ) if $\Omega$ satisfies the assumptions of Theorem 1.9 (exterior domain), then, because of (1.11), $w^{* *}\left(e, u_{0}\right)=2 \sqrt{f^{\prime}(0)}$ for all $e \in \mathbb{S}^{N-1}$ and for all $u_{0} \in \mathcal{E}$;

4) with the same examples as in Theorem 1.13 , there are some domains of $\mathbb{R}^{2}$ satisfying (1.15) for all $e \in \mathbb{S}^{1}$ and such that $w^{* *}\left(e, u_{0}\right)=0$ for all $e \in \mathbb{S}^{1}$ and for all $u_{0} \in \mathcal{E}$

5) given $e \in \mathbb{S}^{N-1}$, there are some domains of $\mathbb{R}^{N}$ satisfying (1.15) and such that $w^{* *}\left(e, u_{0}\right)=+\infty$ for all $u_{0} \in \mathcal{E}$.

In the previous definitions, we chose to consider the leading edge of the region where the reaction starts. It is also of interest to consider the location of the region behind which the reaction is completed. Thus we also introduce the notion of asymptotic spreading speeds, locally uniformly in the direction $e$ or locally along a line $z+\mathbb{R}_{+} e$, of the expanding region where $u$ converges to 1 .

Namely, let $\Omega$ be strongly unbounded in a direction $e \in \mathbb{S}^{N-1}$ and let $u$ solve (1.6) with a given initial condition $u_{0} \in \mathcal{E}$. We assume here that $u(t, x) \rightarrow 1$ as $t \rightarrow+\infty$ locally uniformly in $x \in \bar{\Omega}$. We then define, under the same notation as above,

$$
w_{*}\left(e, u_{0}\right)=\sup \left\{c>0, \forall A>R(e), \limsup _{\substack{(\tau, t) \rightarrow(+\infty,+\infty) \\ \tau \leq c t}}\left[\sup _{\substack{\tau \leq s \leq c t \\ x \in \bar{B}(s e, A)} \bar{\Omega}}|u(t, x)-1|\right]=0\right\}
$$


and, for $z \in \mathbb{R}^{N}$,

$w_{*}\left(e, z, u_{0}\right)=\sup \left\{c>0, \exists A>0, \limsup _{\substack{(\tau, t) \rightarrow(+\infty,+\infty) \\ \tau \leq c t}}\left[\sup _{\substack{\tau \leq s \leq c t \\ x \in \overline{B(z+s e, A)} \cap \bar{\Omega}}}|u(t, x)-1|\right]=0\right\}$.

By convention, we set $w_{*}\left(e, u_{0}\right)=0$ if $u(t, x) \rightarrow 1$ as $t \rightarrow+\infty$ locally uniformly in $x \in \bar{\Omega}$ but if there is no $c>0$ such that, for all $A>R(e), \lim _{\sup _{(\tau, t) \rightarrow(+\infty,+\infty), \tau \leq c t}}$ $\sup _{\tau \leq s \leq c t, x \in \overline{B(s e, A)} \cap \bar{\Omega}}|u(t, x)-1|=0$. We set $w_{*}\left(e, z, u_{0}\right)=0$ if $u(t, x) \rightarrow 1$ as $t \rightarrow+\infty$ locally uniformly in $x \in \bar{\Omega}$ and

$$
\limsup _{\substack{(\tau, t) \rightarrow(+\infty,+\infty) \\ \tau \leq c t}}\left[\sup _{\substack{\tau \in \leq \leq c t \\ x \in \overline{B(z+s e, A)} \cap \bar{\Omega}}}|u(t, x)-1|\right] \neq 0,
$$

for all $c>0$ and $A>0$.

It follows immediately from the above definitions that

$$
w_{*}\left(e, u_{0}\right) \leq w_{*}\left(e, z, u_{0}\right) \leq w^{*}\left(e, z, u_{0}\right) \leq w^{*}\left(e, u_{0}\right)
$$

for all $z \in \mathbb{R}^{N}$ and $u_{0} \in \mathcal{E}$.

If $\Omega$ is a periodic domain satisfying (1.4), then, because of (1.5), the inequalities (1.16) are all equalities for all $e \in \mathbb{R}^{d} \times\{0\}^{N-d}, z \in \mathbb{R}^{N}$ and $u_{0} \in \mathcal{E}$. If $\Omega$ is an exterior domain, all quantities in (1.16) are equal to $2 \sqrt{f^{\prime}(0)}$; see Theorem 1.9 and item 5) below. However, it is an important open problem to determine under which condition on the domain $\Omega$ the equality $w_{*}\left(e, z, u_{0}\right)=w^{*}\left(e, z, u_{0}\right)$ holds. This question is left open.

Furthermore, with the same arguments as the ones used in the next sections, one can prove the following properties:

1 ) if $\Omega$ is locally $C^{2}$ and strongly unbounded in a direction $e \in \mathbb{S}^{N-1}$, then

$$
\forall u_{0} \in \mathcal{E}, \quad w_{*}\left(e, u_{0}\right)=\inf _{z \in \mathbb{R}^{N}} w_{*}\left(e, z, u_{0}\right) ;
$$

2 ) if $\Omega$ is strongly unbounded in a direction $e \in \mathbb{S}^{N-1}$ and locally uniformly of class $C^{2, \alpha}$ with $\alpha>0$, if Hypothesis $H_{y, z}$ is satisfied for some points $y$ and $z$ in $\mathbb{R}^{N}$, and if $u_{0} \in \mathcal{E}$ is less than 1 , then $w_{*}\left(e, y, u_{0}\right)=w_{*}\left(e, z, u_{0}\right)$;

3) if $\Omega$ satisfies the general assumptions of Theorem 1.7, then $w_{*}\left(e, u_{0}\right)$ and $w_{*}\left(e, z, u_{0}\right)$ are nonnegative and do not depend on $u_{0} \in \mathcal{E}$, provided that $u_{0}<1$;

4) if $\Omega$ satisfies the assumptions of Theorem 1.8 (extension property), then, because of (1.10), $w_{*}\left(e, u_{0}\right) \leq w_{*}\left(e, z, u_{0}\right) \leq 2 \sqrt{f^{\prime}(0)}$ for all $u_{0} \in \mathcal{E}, z \in \mathbb{R}^{N}$ and for any direction $e \in \mathbb{S}^{N-1}$ in which $\Omega$ is strongly unbounded;

5 ) if $\Omega$ satisfies the assumptions of Theorem 1.8 (exterior domain), then, because of (1.11), $w_{*}\left(e, u_{0}\right)=w_{*}\left(e, z, u_{0}\right)=2 \sqrt{f^{\prime}(0)}$ for all $e \in \mathbb{S}^{N-1}$, for all $z \in \mathbb{R}^{N}$ and for all $u_{0} \in \mathcal{E}$;

6 ) given $e \in \mathbb{S}^{N-1}$, there are some domains of $\mathbb{R}^{N}$ which are strongly unbounded in the direction $e$ and such that $w_{*}\left(e, u_{0}\right)=w_{*}\left(e, z, u_{0}\right)=+\infty$ for all $u_{0} \in \mathcal{E}$ and $z \in \mathbb{R}^{N}$.

Outline of the paper. The paper is organized as follows: Section 2 is devoted to the proof of the general properties (formula (1.7) in Proposition 1.5 and Theorems 1.6. 1.7, 1.8). Section 3 is concerned with exterior domains (Theorem 1.9) and with the case of domains containing half-cylinders (Proposition 1.11). Section 4 deals with the construction of some domains for which the spreading speeds $w^{*}\left(e, z, u_{0}\right)$ 
really depend on $z$ (the second assertion of Proposition 1.5). We also exhibit in Section 4 some domains with zero or infinite speeds of propagation (Theorem 1.13).

\section{General properties: Dependence on $z$, ON $u_{0}$, AND GENERAL UPPER BOUND}

This section is devoted to the proofs of formula (1.7) and Theorems 1.6, 1.7 and 1.8. More precisely, we prove in Section 2.1 the relationship between the spreading speeds $w^{*}\left(e, u_{0}\right)$ and $w^{*}\left(e, z, u_{0}\right)$. In Section 2.2 , we study the dependence on $u_{0}$. Lastly, in Section 2.3 we prove the general upper bound for the spreading speeds in the large class of domains satisfying the extension property.

2.1. Relationship between $w^{*}\left(e, z, u_{0}\right)$ and $w^{*}\left(e, u_{0}\right)$. Here we first prove the general formula (1.7) in Proposition [1.5 3 We then prove Theorem 1.6 under the asymptotic connectedness assumption $H_{y, z}$.

Proof of formula (1.7) in Proposition 1.5. Let $\Omega \subset \mathbb{R}^{N}$ be strongly unbounded in a given direction $e \in \mathbb{S}^{N-1}$ and let $u_{0} \in \mathcal{E}$ be given. Call $R=R(e)$ the real number defined in Definition 1.1

As already emphasized, the inequality

$$
0 \leq w^{*}\left(e, z, u_{0}\right) \leq w^{*}\left(e, u_{0}\right)
$$

follows from Definitions 1.2 and 1.3, for all $z \in \mathbb{R}^{N}$. Notice also that formula (1.7) is immediate in the case where $w^{*}\left(e, u_{0}\right)=0$. One can then assume here that $w^{*}\left(e, u_{0}\right)>0$. Fix any $\varepsilon \in\left(0, w^{*}\left(e, u_{0}\right)\right)$ and set

$$
\gamma=w^{*}\left(e, u_{0}\right)-\varepsilon .
$$

There exists $A>R$ such that

$$
\sup _{s \geq \gamma t, x \in \overline{B(s e, A)} \cap \bar{\Omega}} u(t, x) \not \rightarrow 0 \text { as } t \rightarrow+\infty .
$$

Therefore, there exist some sequences $\left(t_{n}\right)_{n \in \mathbb{N}} \rightarrow+\infty,\left(s_{n}\right)_{n \in \mathbb{N}}$ such that $s_{n} \geq \gamma t_{n}$, and some points $\left(x_{n}\right)_{n \in \mathbb{N}}$ in $\overline{B_{A}}$ such that $x_{n}+s_{n} e \in \bar{\Omega}$ and

$$
\liminf _{n \rightarrow+\infty} u\left(t_{n}, x_{n}+s_{n} e\right)>0 \text {. }
$$

Up to extraction of some subsequence, one can assume that $x_{n} \rightarrow z \in \overline{B_{A}}$.

We now claim that

$$
w^{*}\left(e, z, u_{0}\right) \geq \gamma
$$

Assume not. Then, owing to Definition 1.3, there is $A^{\prime}>0$ such that

$$
\sup _{s \geq \gamma t, x \in \overline{B_{A^{\prime}}}, x+z+s e \in \bar{\Omega}} u(t, x+z+s e) \rightarrow 0 \text { as } t \rightarrow+\infty .
$$

For $n$ large enough, $x_{n}-z \in \overline{B_{A^{\prime}}}$. On the other hand, $s_{n} \geq \gamma t_{n}$ and $\left(x_{n}-z\right)+z+$ $s_{n} e=x_{n}+s_{n} e \in \bar{\Omega}$. Thus, $u\left(t_{n}, x_{n}+s_{n} e\right) \rightarrow 0$ as $n \rightarrow+\infty$. This contradicts (2.1).

Therefore, the claim $w^{*}\left(e, z, u_{0}\right) \geq \gamma$ is proved. Hence,

$$
w^{*}\left(e, z, u_{0}\right) \geq w^{*}\left(e, u_{0}\right)-\varepsilon
$$

for all $\varepsilon>0$, and formula (1.7) follows.

\footnotetext{
${ }^{3}$ The proof of the second assertion in Proposition 1.5 shall be given in Section 4
} 
Proof of Theorem 1.6. Assume that $\Omega$ is strongly unbounded in the direction $e$, globally of class $C^{2, \alpha}$ for some $\alpha>0$, and that Hypothesis $H_{y, z}$ is satisfied for some points $y$ and $z$ in $\mathbb{R}^{N}$. Under the notation of Hypothesis $H_{y, z}$, there exist $R_{y}>R(e, y), R_{z}>R(e, z), A \geq 0$ and $s_{0}>0$ such that

$$
\forall s \geq s_{0}, \sup _{\substack{y^{\prime} \in \frac{\operatorname{Bu}\left(x+R_{y}\right)}{z^{\prime} \in \bar{\Omega}} \\ z^{\prime} \in \overline{B\left(z+s e, R_{z}\right)} \cap \bar{\Omega}}} d_{\Omega}\left(y^{\prime}, z^{\prime}\right) \leq A .
$$

Notice that this means in particular that

$$
\forall s \geq s_{0}, \overline{B\left(y+s e, R_{y}\right)} \cap \bar{\Omega} \neq \emptyset \text { and } \overline{B\left(z+s e, R_{z}\right)} \cap \bar{\Omega} \neq \emptyset .
$$

Fix any $u_{0}$ in $\mathcal{E}$ and let $u$ solve (1.6). If both spreading speeds $w^{*}\left(e, y, u_{0}\right)$ and $w^{*}\left(e, z, u_{0}\right)$ are infinite, then the desired conclusion $w^{*}\left(e, y, u_{0}\right)=w^{*}\left(e, z, u_{0}\right)$ follows. Assume now that at least one of the spreading speeds, say $w^{*}\left(e, z, u_{0}\right)$, is finite. Fix any $c>w^{*}\left(e, z, u_{0}\right)$. From the above asymptotic connectedness property and from the global smoothness of $\Omega$, Harnack inequality applied in $\bar{\Omega}$ (see [17, 30, 31) yields the existence of $\eta>0$ such that

$$
\frac{\max }{y^{\prime} \in \overline{B\left(y+s e, R_{y}\right)} \cap \bar{\Omega}} u\left(t, y^{\prime}\right) \leq \eta \underset{z^{\prime} \in \overline{B\left(z+s e, R_{z}\right)} \cap \bar{\Omega}}{ } u\left(t+1, z^{\prime}\right) .
$$

On the other hand, owing to Definition 1.3 there exist $c^{\prime} \in\left(w^{*}\left(e, z, u_{0}\right), c\right)$ and $R>0$ such that

$$
\sup _{s^{\prime} \geq c^{\prime} t^{\prime}, z^{\prime} \in \overline{B\left(z+s^{\prime} e, R\right)} \cap \bar{\Omega}} u\left(t^{\prime}, z^{\prime}\right) \rightarrow 0 \text { as } t^{\prime} \rightarrow+\infty .
$$

As already noted in Remark 1.4, one can assume, even if it means decreasing $R$, that $R \leq R_{z}$.

Let $\varepsilon$ be any positive real number. Then there exists $t_{0} \geq 1$ such that

$$
\forall t^{\prime} \geq t_{0}, \forall s^{\prime} \geq c^{\prime} t^{\prime}, \quad \frac{\max }{z^{\prime} \in \overline{B\left(z+s^{\prime} e, R\right)} \cap \bar{\Omega}} u\left(t^{\prime}, z^{\prime}\right) \leq \varepsilon .
$$

One can assume without loss of generality that $t_{0}$ is large enough so that $c t_{0} \geq s_{0}$ and

$$
\forall t \geq t_{0}, c t \geq c^{\prime}(t+1)
$$

This is indeed possible since $c>c^{\prime}$. Now choose any $t \geq t_{0}$ and $s \geq c t$. Observe that $t+1 \geq t_{0}$ and

$$
s \geq c t \geq c^{\prime}(t+1)
$$

whence

$$
\frac{\max }{z^{\prime} \in \overline{B(z+s e, R)} \cap \bar{\Omega}} u\left(t+1, z^{\prime}\right) \leq \varepsilon .
$$

Since $t \geq t_{0} \geq 1$ and $s \geq c t \geq c t_{0} \geq s_{0}$, and since $R \leq R_{z}$, it follows from (2.2) and (2.3) that

$$
\frac{\max }{y^{\prime} \in \overline{B\left(y+s e, R_{y}\right)} \cap \bar{\Omega}} u\left(t, y^{\prime}\right) \leq \eta \underset{z^{\prime} \in \overline{B(z+s e, R)} \cap \bar{\Omega}}{\min } u\left(t+1, z^{\prime}\right) \leq \eta \varepsilon .
$$

Since this is true for all $t \geq t_{0}$ and $s \geq c t$ and since $\eta$ is independent of $\varepsilon$, one obtains

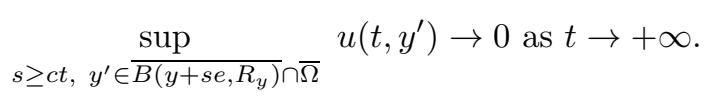

Therefore, $w^{*}\left(e, y, u_{0}\right)$ is finite and $w^{*}\left(e, y, u_{0}\right) \leq c$. Since this inequality holds for all $c>w^{*}\left(e, z, u_{0}\right)$, one obtains $w^{*}\left(e, y, u_{0}\right) \leq w^{*}\left(e, z, u_{0}\right)$. 
By changing the role of $y$ and $z$, one then concludes that $w^{*}\left(e, y, u_{0}\right)=w^{*}\left(e, z, u_{0}\right)$, and the proof of Theorem 1.6 is complete.

Remark 2.1. We prove here that if $\Omega$ is strongly unbounded in a direction $e \in \mathbb{S}^{N-1}$ and if Hypothesis $H_{y, z}$ is satisfied for some $y, z \in \mathbb{R}^{N}$, then so is $H_{y+\alpha e, z+\beta e}$ for any $(\alpha, \beta) \in \mathbb{R}^{2}$. Assume $H_{y, z}$ and let $(\alpha, \beta) \in \mathbb{R}^{2}$ be given. Then there exist $A \geq 0$, $s_{0}>0$ and some radii $R_{y}>R_{y}^{\prime}>R(e, y)$ and $R_{z}>R_{z}^{\prime}>R(e, z)$ such that

$\forall s \geq s_{0}, \quad\left\{\begin{array}{l}\overline{B\left(y+s e, R_{y}\right)} \cap \bar{\Omega} \supset \overline{B\left(y+s e, R_{y}^{\prime}\right)} \cap \bar{\Omega} \neq \emptyset, \\ \overline{B\left(z+s e, R_{z}\right)} \cap \bar{\Omega} \supset \overline{B\left(z+s e, R_{z}^{\prime}\right)} \cap \bar{\Omega} \neq \emptyset, \\ \forall y^{\prime} \in \overline{B\left(y+s e, R_{y}\right)} \cap \bar{\Omega}, \forall z^{\prime} \in \overline{B\left(z+s e, R_{z}\right)} \cap \bar{\Omega}, \quad d_{\Omega}\left(y^{\prime}, z^{\prime}\right) \leq A .\end{array}\right.$

As a consequence,

$$
\forall s \geq s_{0}, \forall y^{\prime}, y^{\prime \prime} \in \overline{B\left(y+s e, R_{y}\right)} \cap \bar{\Omega}, \quad d_{\Omega}\left(y^{\prime}, y^{\prime \prime}\right) \leq 2 A .
$$

Let $n \in \mathbb{N} \backslash\{0\}$ be such that $\varepsilon=\alpha / n$ satisfies $|\varepsilon| \leq R_{y}-R_{y}^{\prime}$. It follows that, for all $k=0, \ldots, n-1$ and for all $s \geq s_{0}+|\alpha|$, there holds

$\overline{B\left(y+s e+k \varepsilon e, R_{y}\right)} \cap \overline{B\left(y+s e+(k+1) \varepsilon e, R_{y}\right)} \cap \bar{\Omega} \supset \overline{B\left(y+s e+k \varepsilon e, R_{y}^{\prime}\right)} \cap \bar{\Omega} \neq \emptyset$.

Therefore, for any $s \geq s_{0}+|\alpha|, y^{\prime} \in \overline{B\left(y+s e, R_{y}\right)} \cap \bar{\Omega}$ and $y^{\prime \prime} \in \overline{B\left(y+s e+\alpha e, R_{y}\right)} \cap$ $\bar{\Omega}$, there is a sequence of points $\left(y_{k}\right)_{0 \leq k \leq n-1}$ such that

$$
\forall k=0, \ldots, n-1, \quad y_{k} \in \overline{B\left(y+s e+k \varepsilon e, R_{y}\right)} \cap \overline{B\left(y+s e+(k+1) \varepsilon e, R_{y}\right)} \cap \bar{\Omega},
$$

whence

$d_{\Omega}\left(y^{\prime}, y^{\prime \prime}\right) \leq d_{\Omega}\left(y^{\prime}, y_{0}\right)+d_{\Omega}\left(y_{0}, y_{1}\right)+\cdots+d_{\Omega}\left(y_{n-2}, y_{n-1}\right)+d_{\Omega}\left(y_{n-1}, y^{\prime \prime}\right) \leq 2 A(n+1)$

from (2.4). As a consequence,

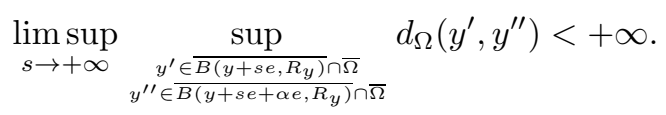

The same conclusion holds with $\left(z, R_{z}, \beta\right)$ instead of $\left(y, R_{y}, \alpha\right)$. As a conclusion,

$$
\limsup _{s \rightarrow+\infty} \frac{\sup }{\substack{y^{\prime} \in \overline{B\left(y+s e+\alpha e, R_{y}\right)} \cap \bar{\Omega} \\ z^{\prime} \in \bar{B}\left(z+s e+\beta e, R_{z}\right) \cap \bar{\Omega}}} d_{\Omega}\left(y^{\prime}, z^{\prime}\right)<+\infty ;
$$

that is, Hypothesis $H_{y+\alpha e, z+\beta e}$ is satisfied.

2.2. Dependence on the initial datum. The proof of Theorem 1.7 is based on some auxiliary results. Let us first introduce some notation. If $D$ is an open subset of $\mathbb{R}^{N}$ such that $\bar{\Omega} \cap D \neq \emptyset$, we say

$$
\lambda_{D}=\inf _{\psi \in C_{c}^{1}(\bar{\Omega} \cap D), \psi \neq 0} \frac{\int_{\Omega \cap D}|\nabla \psi|^{2}}{\int_{\Omega \cap D} \psi^{2}},
$$

where $C_{c}^{1}(\bar{\Omega} \cap D)$ denotes the set of functions which are of class $C^{1}$ in $\bar{\Omega} \cap D$ and have a support which is compactly included in $\bar{\Omega} \cap D$. It follows immediately that the map $D \mapsto \lambda_{D}$ is nonincreasing with respect to the inclusion (in the class of open sets $D$ such that $\bar{\Omega} \cap D \neq \emptyset$ ). 
Note that the definition of $\lambda_{D}$ heuristically corresponds to the principal eigenvalue problem with mixed boundary conditions:

$$
\left\{\begin{aligned}
-\Delta \psi & =\lambda_{D} \psi & & \text { in } \Omega \cap D, \\
\psi & =0 & & \text { on } \partial D \cap \Omega, \\
\nu \cdot \nabla \psi & =0 & & \text { on } \partial \Omega \cap D .
\end{aligned}\right.
$$

However, since no regularity on $\Omega \cap \bar{D}$ is assumed, the meaning of (2.6) is somewhat delicate. Hence, this is only formal. Therefore, we prefer to deal directly with the definition (2.5) of $\lambda_{D}$ rather than with the eigenvalue problem (2.6).

Under the assumptions of Theorem 1.7, for all $r \geq R_{0}$ and $z \in \mathbb{R}^{N}$, we say

$$
\lambda_{r}^{z}=\lambda_{B(z, r)},
$$

where we recall that $B(z, r)$ denotes the open euclidean ball of radius $r$ and centre $z$.

Lemma 2.2. Under the assumptions of Theorem 1.7,

$$
\lambda_{r}^{z} \rightarrow 0 \text { as } r \rightarrow+\infty \text { uniformly in } z \in \mathbb{R}^{N} .
$$

Proof. Fix a family $\left(\zeta_{r}\right)_{r \geq R_{0}}$ of $C^{\infty}\left(\mathbb{R}^{N}\right)$ functions such that, for each $r \geq R_{0}$, the support of $\zeta_{r}$ is included in $B(0, r+1)$ and $\zeta_{r}=1$ in $B(0, r)$. One can choose the functions $\zeta_{r}$ so that

$$
\left\|\zeta_{r}\right\|_{C^{1}(B(0, r+1))}=\left\|\zeta_{r}\right\|_{\infty}+\left\|\nabla \zeta_{r}\right\|_{\infty} \leq C
$$

where $C$ is a positive constant which is independent of $r \geq R_{0}$.

Let $r \geq R_{0}$ and let $z$ be any point in $\mathbb{R}^{N}$. Call $\zeta_{r}^{z}$ the function defined by $\zeta_{r}^{z}(x)=\zeta_{r}(x-z)$ for all $x \in \mathbb{R}^{N}$. One has

$$
0 \leq \lambda_{r+1}^{z} \leq \frac{\int_{\Omega \cap B(z, r+1)}\left|\nabla \zeta_{r}^{z}\right|^{2}}{\int_{\Omega \cap B(z, r+1)}\left(\zeta_{r}^{z}\right)^{2}} \leq C^{2} \frac{|\Omega \cap(B(z, r+1) \backslash B(z, r))|}{|\Omega \cap B(z, r)|}=C^{2} \frac{\mu_{r+1}^{z}-\mu_{r}^{z}}{\mu_{r}^{z}},
$$

where $|E|$ denotes the Lebesgue measure of $E \subset \mathbb{R}^{N}$. Since $\mu_{r+1}^{z} / \mu_{r}^{z} \rightarrow 1$ uniformly in $z \in \mathbb{R}^{N}$ as $r \rightarrow+\infty$, the conclusion of Lemma 2.2 follows.

Since the proof of Theorem 1.7 is somewhat involved, we start with a simpler case which is of independent interest. Even though it can be seen to be a consequence of Theorem 1.7 here we include the proof, since the ideas will be clearer in the elliptic case than in the parabolic case which involves several technical difficulties.

Theorem 2.3. Let $\Omega$ be a domain satisfying the assumptions of Theorem 1.7, Let $g:[0,+\infty) \rightarrow \mathbb{R}$ be a $C^{1}$ function such that $g(0)=g(1)=0, g^{\prime}(0)>0, g>0$ in $(0,1)$ and $g<0$ in $(1,+\infty)$. Let $u$ be a classical bounded solution of

$$
\left\{\begin{aligned}
\Delta u+g(u)=0 & & \text { in } \bar{\Omega}, \\
u \geq 0 & & \text { in } \bar{\Omega}, \\
\nu \cdot \nabla u=0 & & \text { on } \partial \Omega .
\end{aligned}\right.
$$

Then $u \equiv 0$ or $u \equiv 1$.

Proof. Assume that $u \not \equiv 0$. Then $u>0$ in $\bar{\Omega}$ from the strong maximum principle and the Hopf lemma. We first prove that

$$
m:=\inf _{\bar{\Omega}} u>0 .
$$


Assume not. Under the notation of Theorem 1.7. Lemma 2.2 yields the existence of $R \geq R_{0}$ such that

$$
\forall z \in \mathbb{R}^{N}, \lambda_{R}^{z}<\frac{g^{\prime}(0)}{2} .
$$

Then there exists a sequence of points $\left(z_{n}\right)_{n \in \mathbb{N}}$ in $\bar{\Omega}$ such that $u\left(z_{n}\right) \rightarrow 0$ as $n \rightarrow$ $+\infty$. From the connectedness assumption (1.8) and the global smoothness of $\Omega$, Harnack inequality applied in $\bar{\Omega}$ implies that

$$
\max _{\left|z-z_{n}\right| \leq R, z \in \bar{\Omega}} u(z) \rightarrow 0 \text { as } n \rightarrow+\infty .
$$

Therefore, there is $M \in \mathbb{N}$ such that

$$
0 \geq \Delta u+\frac{g^{\prime}(0)}{2} u \text { in } \overline{B\left(z_{M}, R\right)} \cap \bar{\Omega} .
$$

Since $\lambda_{R}^{z_{M}}<g^{\prime}(0) / 2$, there is a function $w \in C_{c}^{1}\left(\bar{\Omega} \cap B\left(z_{M}, R\right)\right)$ such that $w \not \equiv 0$ and

$$
\frac{\int_{\Omega \cap B\left(z_{M}, R\right)}|\nabla w|^{2}}{\int_{\Omega \cap B\left(z_{M}, R\right)} w^{2}}<\frac{g^{\prime}(0)}{2} .
$$

Multiply (2.9) by $w^{2} / u \geq 0$ and integrate by parts over $\Omega \cap B\left(z_{M}, R\right)$. One can think of $w$ in (2.10) as an approximation of an eigenfunction of (2.6). Since $\nu \cdot \nabla u=0$ on $\partial \Omega$ and $w \in C_{c}^{1}\left(\bar{\Omega} \cap B\left(z_{M}, R\right)\right)$, one obtains

$$
\begin{aligned}
0 & \geq \frac{g^{\prime}(0)}{2} \int_{\Omega \cap B\left(z_{M}, R\right)} w^{2}-\int_{\Omega \cap B\left(z_{M}, R\right)} \nabla u \cdot \nabla\left(\frac{w^{2}}{u}\right) \\
& =\frac{g^{\prime}(0)}{2} \int_{\Omega \cap B\left(z_{M}, R\right)} w^{2}-2 \int_{\Omega \cap B\left(z_{M}, R\right)} \frac{w \nabla u \cdot \nabla w}{u}+\int_{\Omega \cap B\left(z_{M}, R\right)} \frac{w^{2}|\nabla u|^{2}}{u^{2}} \\
& \geq \frac{g^{\prime}(0)}{2} \int_{\Omega \cap B\left(z_{M}, R\right)} w^{2}-\int_{\Omega \cap B\left(z_{M}, R\right)}^{|\nabla w|^{2} .}
\end{aligned}
$$

The last inequality contradicts property (2.10).

Hence, the claim (2.8) holds. Now choose $\xi_{0}$ such that

$$
0<\xi_{0}<\min (m, 1),
$$

and let $\xi(t)$ be the solution of $\dot{\xi}(t)=g(\xi(t))$ with $\xi(0)=\xi_{0}$. Since $g>0$ on $(0,1)$ and $g(1)=0$, one obtains $\dot{\xi}(t)>0$ for all $t \geq 0$ and $\xi(+\infty)=1$. On the other hand, since $u$ solves (2.7), the parabolic maximum principle implies that $u(z) \geq \xi(t)$ for all $z \in \bar{\Omega}$ and $t \geq 0$. Thus, $m \geq 1$.

Similarly, using the fact that $g<0$ in $(1,+\infty)$, one obtains $\sup _{\bar{\Omega}} u \leq 1$. As a conclusion, $u \equiv 1$, and the proof of Theorem 2.3 is complete.

Remark 2.4. It can be seen (in the case $\Omega=\mathbb{R}^{N}$, without the boundary conditions) that the conclusion of Theorem 2.3 is not true without the boundedness assumption. For instance, let $g$ be any $C^{1}([0,+\infty))$ function such that $g(s)=1-s$ for all $s \geq 1$. Then, for any unit vector $\alpha \in \mathbb{R}^{N}$, the function $w=1+e^{\alpha \cdot x}$ is a nonnegative unbounded solution of $\Delta w+g(w)=0$ in $\mathbb{R}^{N}$. 
Proof of Theorem 1.7. Let $u$ be a solution of (1.6) with a given initial condition $u_{0} \in \mathcal{E}$. We shall prove here that $u(t, x) \rightarrow 1$ uniformly locally in $x \in \bar{\Omega}$ as $t \rightarrow+\infty$. The proof is much more delicate than the elliptic analogue. The reason is that the time at which the solution $u$ will be above a suitably chosen positive number in any given compact set does depend on the compact set.

As above, since $u_{0}$ is bounded, there is $M>1$ such that $u_{0} \leq M$ in $\bar{\Omega}$. Hence

$$
u(t, x) \leq \zeta(t)
$$

for all $t \geq 0$ and $x \in \bar{\Omega}$, where $\zeta$ solves $\dot{\zeta}(t)=f(\zeta(t))$ with $\zeta(0)=M$. Since $f$ is negative in $(1,+\infty)$ and vanishes at 1 , one has that $1 \leq \zeta(t) \leq M$ for all $t \geq 0$ and $\zeta(t) \rightarrow 1$ as $t \rightarrow+\infty$. Hence $u(t, x) \leq M$ for all $t \geq 0$ and $x \in \bar{\Omega}$, and

$$
\limsup _{t \rightarrow+\infty}\left(\sup _{x \in \bar{\Omega}} u(t, x)\right) \leq 1 .
$$

Let us now prove a lower estimate. To do so, choose $R \geq R_{0}$ such that

$$
\forall z \in \mathbb{R}^{N}, \lambda_{R}^{z}<\frac{f^{\prime}(0)}{2} .
$$

This is possible thanks to Lemma 2.2. Owing to the choices of $R$ and $R_{0}$, one knows that $\Omega \cap B(z, R) \neq \emptyset$ for all $z \in \mathbb{R}^{N}$. Furthermore, since $\Omega$ is globally smooth and satisfies (1.8), Harnack inequality applied in $\bar{\Omega}$ yields the existence of a constant $C>0$ such that, for any solution $v$ of (1.6) with an initial condition $v_{0} \in \mathcal{E}$ satisfying $v_{0} \leq 1$, the following inequality holds:

$$
\forall t \geq 1, \forall z \in \mathbb{R}^{N}, \min _{\bar{\Omega} \cap \overline{B(z, R)}} v(t+1, \cdot) \geq C_{\bar{\Omega} \cap \frac{\max }{B(z, R)}} v(t, \cdot) .
$$

Notice that all such solutions $v$ satisfy $0 \leq v(t, x) \leq 1$ for all $t \geq 0$ and $x \in \bar{\Omega}$.

Also let $s_{0} \in(0,1)$ be such that

$$
\forall s \in\left[0, s_{0}\right], f(s) \geq \frac{f^{\prime}(0)}{2} s .
$$

Since $u(1, \cdot)$ is a positive continuous function in $\bar{\Omega}$, there exists $v_{0} \in \mathcal{E}$ such that

$$
\operatorname{supp}\left(v_{0}\right) \subset \bar{\Omega} \cap \overline{B(0, R)} \text { and } v_{0}(x) \leq \min \left(1, C s_{0}, u(1, x)\right) \text { in } \bar{\Omega},
$$

where $\operatorname{supp}\left(v_{0}\right)$ denotes the support of $v_{0}$. Notice that $0<v(t, x)<1<M$ for all $t>0$ and $x \in \bar{\Omega}$ from the strong parabolic maximum principle.

We now claim that

$$
\forall z_{0} \in \mathbb{R}^{N}, \exists t_{z_{0}} \geq 2, v\left(t_{z_{0}}, \cdot\right) \geq C s_{0} \text { in } \bar{\Omega} \cap \overline{B\left(z_{0}, R\right)} .
$$

Assume that this is not the case for some point $z_{0} \in \mathbb{R}^{N}$. From (2.12), it then follows that

$$
\forall t \geq 1, \max _{\bar{\Omega} \cap \overline{B\left(z_{0}, R\right)}} v(t, \cdot) \leq s_{0} .
$$

In view of the choice of $s_{0}$, we know that

$$
\forall t \geq 1, \forall x \in \bar{\Omega} \cap \overline{B\left(z_{0}, R\right)}, v_{t}(t, x) \geq \Delta v(t, x)+\frac{f^{\prime}(0)}{2} v(t, x) .
$$

Since $\lambda_{R}^{z_{0}}<f^{\prime}(0) / 2$, there is a function $w \in C_{c}^{1}\left(\bar{\Omega} \cap B\left(z_{0}, R\right)\right)$ such that $w \not \equiv 0$ and

$$
\int_{\Omega \cap B\left(z_{0}, R\right)}|\nabla w|^{2}<\frac{f^{\prime}(0)}{2} \times \int_{\Omega \cap B\left(z_{0}, R\right)} w^{2} .
$$


For each fixed $t \geq 1$, set

$$
\Theta(t)=\int_{\Omega \cap B\left(z_{0}, R\right)} w^{2}(x) \ln v(t, x) d x .
$$

Multiply (2.15) by $w^{2} / v(t, \cdot) \geq 0$ and integrate by parts over $\Omega \cap B\left(z_{0}, R\right)$. The same calculations as in Theorem 2.3 then give

$$
\forall t \geq 1, \Theta^{\prime}(t) \geq \frac{f^{\prime}(0)}{2} \times \int_{\Omega \cap B\left(z_{0}, R\right)} w^{2}-\int_{\Omega \cap B\left(z_{0}, R\right)}|\nabla w|^{2} .
$$

From the choice of $w$, the right-hand side of (2.16) is a positive number which is independent of $t$. Therefore, $\Theta(t) \rightarrow+\infty$ as $t \rightarrow+\infty$. However,

$$
\Theta(t) \leq\left(\ln s_{0}\right) \times \int_{\Omega \cap B\left(z_{0}, R\right)} w^{2}(x) d x
$$

for all $t \geq 1$, thanks to (2.14). One has then reached a contradiction.

Therefore, the claim (2.13) has been proved. Now, from Harnack inequality applied to $v$ in $\bar{\Omega}$, there exists a constant $C^{\prime}>0$ such that

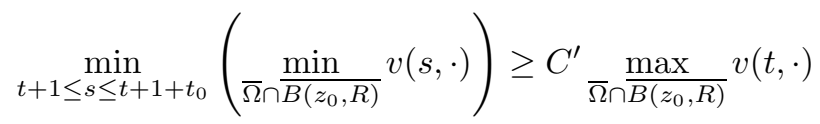

for all $t \geq 2$ and $z_{0} \in \mathbb{R}^{N}$. On the other hand, from the choice of $v_{0}$ and from (2.13) applied at $z_{0}=0$, it follows that

$$
v\left(t_{0}, \cdot\right) \geq v_{0} \text { in } \bar{\Omega} .
$$

Hence $v\left(t+t_{0}, x\right) \geq v(t, x)$ for all $t \geq 0$ and $x \in \bar{\Omega}$ from the parabolic maximum principle. As a consequence, from (2.13) we infer that

$$
\bar{\Omega} \cap \overline{\max } \frac{}{B\left(z_{0}, R\right)} v\left(t_{z_{0}}+k t_{0}, \cdot\right) \geq \min _{\bar{\Omega} \cap \overline{B\left(z_{0}, R\right)}} v\left(t_{z_{0}}+k t_{0}, \cdot\right) \geq C s_{0}
$$

for all $k \in \mathbb{N}$ and for all $z_{0} \in \mathbb{R}^{N}$. Together with (2.17), one obtains

$$
\min _{\bar{\Omega} \cap \overline{B\left(z_{0}, R\right)}} v(t, \cdot) \geq C^{\prime} C s_{0}
$$

for all $t \geq t_{z_{0}}+1$ and for all $z_{0} \in \mathbb{R}^{N}$.

Lastly, $u(t+1, x) \geq v(t, x)$ for all $t \geq 0$ and $x \in \bar{\Omega}$ from the choice of $v_{0}$. Therefore,

$$
\forall z_{0} \in \mathbb{R}^{N}, \forall t \geq t_{z_{0}}+2, \underset{\bar{\Omega} \cap \overline{B i n}}{B\left(z_{0}, R\right)} u(t, \cdot) \geq C^{\prime} C s_{0}>0,
$$

where we recall that the positive constants $C, C^{\prime}$ and $s_{0}$ do not depend on the point $z_{0}$. Now pick any sequence $\left(t_{n}\right)_{n \in \mathbb{N}}$ of positive real numbers such that $t_{n} \rightarrow+\infty$ as $n \rightarrow+\infty$. Recall that $0 \leq u(t, x) \leq M$ for all $t \geq 0$ and $x \in \bar{\Omega}$. From standard parabolic estimates, up to extraction of a subsequence, the functions

$$
u_{n}(t, x)=u\left(t+t_{n}, x\right)
$$

converge locally uniformly in $(t, x) \in \mathbb{R} \times \bar{\Omega}$ to a classical nonnegative solution $U$ of

$$
\left\{\begin{aligned}
U_{t} & =\Delta U+f(U) & & \text { in } \Omega, t \in \mathbb{R} \\
\nu \cdot \nabla U & =0 & & \text { on } \partial \Omega, t \in \mathbb{R} .
\end{aligned}\right.
$$

One knows that $U(t, x) \leq 1$ for all $(t, x) \in \mathbb{R} \times \bar{\Omega}$ from (2.11). Furthermore,

$$
U(t, x) \geq C^{\prime} C s_{0}>0 \text { for all }(t, x) \in \mathbb{R} \times \bar{\Omega}
$$


from (2.18). Notice that this implies in particular that $0<C C^{\prime} s_{0} \leq 1$. Now call $\omega(t)$ the solution of $\dot{\omega}(t)=f(\omega(t))$ for all $t \geq 0$ with $\omega(0)=C C^{\prime} s_{0}$. Since $f>0$ in $(0,1)$ and $f(1)=0$, one has $\omega(t) \rightarrow 1$ as $t \rightarrow+\infty$. For any $t \in \mathbb{R}$ and $T>0$, since $U(t-T, \cdot) \geq C C^{\prime} s_{0}$ in $\bar{\Omega}$, the maximum principle implies that $U(t, \cdot) \geq \omega(T)$ in $\bar{\Omega}$. Since this holds for all $t \in \mathbb{R}$ and $T>0$, one concludes that $U(t, x) \geq 1$ for all $(t, x) \in \mathbb{R} \times \bar{\Omega}$. Eventually,

$$
U=1 \text { in } \mathbb{R} \times \bar{\Omega},
$$

and by uniqueness of the limit, it follows that

$$
u(t, x) \rightarrow 1
$$

locally uniformly in $x \in \bar{\Omega}$ as $t \rightarrow+\infty$.

Now let $u_{0}$ and $v_{0}$ be two continuous, nonnegative and nonzero functions which are compactly supported in $\bar{\Omega}$. Assume that $u_{0}$ and $v_{0}$ are less than 1 . Let $e$ be a unit vector in $\mathbb{R}^{N}$. Notice that the assumptions in Theorem 1.7 readily imply that $\Omega$ is strongly unbounded in the direction $e$. Since $\max _{\bar{\Omega}} v_{0}<1$ and $v_{0}$ is compactly supported, it follows from the first part of the proof of Theorem 1.7 that

$$
u\left(t_{0}, x\right) \geq v_{0}(x)
$$

for all $x \in \bar{\Omega}$, for some $t_{0} \geq 0$. Therefore, $u\left(t+t_{0}, x\right) \geq v(t, x)$ for all $t \geq 0$ and $x \in \bar{\Omega}$, whence $w^{*}\left(e, u_{0}\right) \geq w^{*}\left(e, v_{0}\right)$.

Changing the roles of $u$ and $v$ leads to the inequality $w^{*}\left(e, v_{0}\right) \geq w^{*}\left(e, u_{0}\right)$. Therefore, $w^{*}\left(e, u_{0}\right)=w^{*}\left(e, v_{0}\right)$.

The same arguments also imply that

$$
w^{*}\left(e, z, u_{0}\right)=w^{*}\left(e, z, v_{0}\right)
$$

for all $e \in \mathbb{S}^{N-1}, z \in \mathbb{R}^{N}$ and $\left(u_{0}, v_{0}\right) \in \mathcal{E}^{2}$ with $u_{0}, v_{0}<1$ in $\bar{\Omega}$. The proof of Theorem 1.7 is thus complete.

2.3. Upper bound for domains with the extension property. This section is devoted to the proof of Theorem 1.8. To do so, we first state a general upper bound for the heat kernel in domains satisfying the extension property. This upper bound follows from general results of Davies [10] and Grigor'yan [16].

Proposition 2.5. Let $\Omega$ be a locally $C^{2}$ nonempty connected open subset of $\mathbb{R}^{N}$ satisfying the extension property. Call $p(t, \cdot, \cdot)$ the heat kernel in $\bar{\Omega}$ with Neumann boundary condition on $\partial \Omega$. Then for any $C_{0}>4$, there exist two positive constants $C$ and $\delta$ such that

$$
\forall t>0, \forall(z, x) \in \bar{\Omega} \times \bar{\Omega}, p(t, z, x) \leq C \times\left(1+(\delta t)^{-N / 2}\right) \times \exp \left[-\frac{d_{\Omega}(z, x)^{2}}{C_{0} t}\right],
$$

where $d_{\Omega}$ denotes the geodesic distance in $\bar{\Omega}$.

Proof. Let $C_{0}>4$ be given. Since $\Omega$ satisfies the extension property, it follows from Theorem 2.4.4 by Davies [10] that there exists $C_{1}>0$ such that $0 \leq p(t, z, x) \leq$ $C_{1} t^{-N / 2}$ for all $0<t \leq 1$ and for all $(z, x) \in \bar{\Omega} \times \bar{\Omega}$. The maximum principle then yields $p(t, z, x) \leq C_{1}$ for all $t \geq 1$ and $(z, x) \in \bar{\Omega} \times \bar{\Omega}$. In particular,

$$
\forall t>0, \forall x \in \Omega, \quad p(t, x, x) \leq \frac{1}{g(t)},
$$

where $g(t)=\left(C_{1} t^{-N / 2}+C_{1}\right)^{-1}$. 
The function $g$ is "regular" in the sense of [16], and, since $C_{0}>4$, it follows from the Gaussian upper bounds by Grigor'yan [16] that there exist two positive constants $\delta$ and $C_{2}$, which only depend on $C_{0}$ and $g$, such that

$$
\forall t>0, \forall(z, x) \in \bar{\Omega} \times \bar{\Omega}, \quad p(t, z, x) \leq \frac{C_{2}}{g(\delta t)} \exp \left[-\frac{d_{\Omega}(z, x)^{2}}{C_{0} t}\right]
$$

The conclusion of Proposition 2.5 follows with $C=C_{1} C_{2}$.

Proof of Theorem 1.8, As already mentioned, it is sufficient to prove property (1.10). Fix a speed $c>2 \sqrt{f^{\prime}(0)}$ and $u_{0} \in \mathcal{E}$. Then let $R_{0}>0$ be such that $B_{R_{0}}$ contains the support of $u_{0}$ and let $C_{0}>4, \varepsilon>0$ and $t_{0}>0$ be such that

$$
\forall t \geq t_{0}, \forall z \in B_{R_{0}}, \forall|x| \geq c t, \quad \frac{|z-x|^{2}}{C_{0} t} \geq\left(f^{\prime}(0)+\varepsilon\right) t .
$$

Call $v(t, x)$ the solution of

$$
\left\{\begin{aligned}
v_{t} & =\Delta v \\
\nu \cdot \nabla v & =0 \text { on } \partial \Omega
\end{aligned}\right.
$$

with initial condition $u_{0}$. Since $f(s) \leq f^{\prime}(0) s$ for all $s \geq 0$, the maximum principle yields

$$
0 \leq u(t, x) \leq v(t, x) \times \exp \left(f^{\prime}(0) t\right)
$$

for all $t \geq 0$ and $x \in \bar{\Omega}$.

The function $v$ can be written as

$$
v(t, x)=\int_{\Omega} p(t, z, x) u_{0}(z) d z=\int_{B_{R_{0}}} p(t, z, x) u_{0}(z) d z .
$$

From Proposition 2.5, there exist two positive constants $C$ and $\delta$ such that

$$
v(t, x) \leq C\left\|u_{0}\right\|_{\infty} \times\left(1+(\delta t)^{-N / 2}\right) \times \int_{B_{R_{0}}} \exp \left[-\frac{d_{\Omega}(z, x)^{2}}{C_{0} t}\right] d z
$$

for all $t>0$ and $x \in \bar{\Omega}$. Therefore,

$$
0 \leq u(t, x) \leq C \exp \left(f^{\prime}(0) t\right)\left\|u_{0}\right\|_{\infty} \times\left(1+(\delta t)^{-N / 2}\right) \times \int_{B_{R_{0}}} \exp \left[-\frac{|z-x|^{2}}{C_{0} t}\right] d z
$$

for all $t>0$ and $x \in \bar{\Omega}$. One concludes from (2.20) that

$$
0 \leq u(t, x) \leq C\left\|u_{0}\right\|_{\infty} \times\left(1+(\delta t)^{-N / 2}\right) \times\left|B_{R_{0}}\right| \exp (-\varepsilon t)
$$

for all $t \geq t_{0}$ and $|x| \geq c t, x \in \bar{\Omega}$ (remember that $\left|B_{R_{0}}\right|$ denotes the Lebesgue measure of the ball $\left.B_{R_{0}}\right)$. The estimate (1.10) follows, whence

$$
w^{*}\left(e, z, u_{0}\right) \leq w^{*}\left(e, u_{0}\right) \leq 2 \sqrt{f^{\prime}(0)}
$$

for all $z \in \mathbb{R}^{N}$ and $u_{0} \in \mathcal{E}$. 


\section{ExTERIOR DOMAINS AND DOMAINS CONTAINING LARGE HALF-CYLINDERS}

This section is devoted to the proofs of Theorem [1.9] and Proposition 1.11. We first deal with the case of exterior domains.

Lemma 3.1. Let $\Omega$ be an exterior domain of $\mathbb{R}^{N}$ of class $C^{2}$, let $u_{0} \not \equiv 0$ be nonnegative, continuous, bounded in $\bar{\Omega}$ and let $u(t, x)$ be the solution of (1.6) with initial condition $u_{0}$. Assume that $f: \mathbb{R}_{+} \rightarrow \mathbb{R}$ is $C^{1}$ and such that $f(0)=f(1)=0$, $f^{\prime}(0)>0, f>0$ on $(0,1)$ and $f<0$ on $(1,+\infty)$. Then $u(t, x) \rightarrow 1$ locally uniformly in $x \in \bar{\Omega}$ as $t \rightarrow+\infty$.

If $\Omega$ were smoother (of class $C^{2, \alpha}$ ), then Lemma 3.1 would follow from Theorem 1.7. The proof of Lemma 3.1 is actually simpler than that of the first part of Theorem 1.7.

Proof of Lemma 3.1. First of all, as in the proof of Theorem 1.7, it follows from the boundedness of $u_{0}$ and from the profile of $f$ that

$$
\limsup _{t \rightarrow+\infty}\left(\sup _{x \in \bar{\Omega}} u(t, x)\right) \leq 1 .
$$

Choose $R>0$ large enough so that $\lambda_{R}<f^{\prime}(0)$, where $\left(\lambda_{R}, \psi_{R}\right)$ is the pair of the first eigenvalue and the first eigenfunction of the problem

$$
\left\{\begin{aligned}
-\Delta \psi_{R} & =\lambda_{R} \psi_{R} & & \text { in } B_{R}, \\
\psi_{R} & >0 & & \text { in } B_{R}, \\
\psi_{R} & =0 & & \text { on } \partial B_{R} \\
\left\|\psi_{R}\right\|_{L^{\infty}\left(B_{R}\right)} & =1 . & &
\end{aligned}\right.
$$

This is indeed possible since $\lambda_{\rho} \rightarrow 0$ as $\rho \rightarrow+\infty$.

Then fix $R_{0}>0$ such that

$$
\mathbb{R}^{N} \backslash \Omega \subset B_{R_{0}}
$$

From the strong parabolic maximum principle, one has $u(t, x)>0$ for all $t>0$ and $x \in \bar{\Omega}$. Therefore, by continuity, there exists $\varepsilon \in(0,1)$ such that

$$
u(1, x) \geq \varepsilon \text { for all } x \text { such that } R_{0} \leq|x| \leq R_{0}+2 R .
$$

Even if it means decreasing $\varepsilon>0$, one can assume from the choice of $R$ that

$$
\Delta\left(\varepsilon \psi_{R}\right)+f\left(\varepsilon \psi_{R}\right)=-\varepsilon \lambda_{R} \psi_{R}+f\left(\varepsilon \psi_{R}\right) \geq 0 \text { in } B_{R}
$$

Since

$$
u(1, x) \geq \varepsilon \geq \varepsilon \psi_{R}\left(x-x_{0}\right) \text { for all } x \in \overline{B\left(x_{0}, R\right)}
$$

and for all $x_{0} \in \mathbb{R}^{N}$ such that $\left|x_{0}\right|=R_{0}+R$, it follows that

$$
u(1+t, x) \geq v(t, x) \text { for all } t \geq 0 \text { and for all } x \in \bar{\Omega},
$$

where $v$ is the solution of (1.6) with initial condition $v_{0}(x)=\max _{\left|x_{0}\right|=R_{0}+R} v_{0, x_{0}}(x)$ defined by

$$
v_{0, x_{0}}(x)= \begin{cases}\varepsilon \psi_{R}\left(x-x_{0}\right) & \text { if } x \in \overline{B\left(x_{0}, R\right)} \\ 0 & \text { if } x \in \bar{\Omega} \backslash \overline{B\left(x_{0}, R\right)}\end{cases}
$$

The function $v_{0}$ is a subsolution for the associated elliptic equation and $v(t, x)$ is then nondecreasing with respect to $t$. Moreover, $v_{0} \leq 1$ in $\bar{\Omega}$, whence $v(t, x) \leq 1$ 
for all $t \geq 0$ and $x \in \bar{\Omega}$. Hence, standard parabolic estimates imply that $v(t, x)$ converges locally uniformly in $x \in \bar{\Omega}$ as $t \rightarrow+\infty$ to a classical solution $v_{\infty}$ of

$$
\left\{\begin{array}{rll}
\Delta v_{\infty}+f\left(v_{\infty}\right) & =0 \text { in } \Omega \\
\nu \cdot \nabla v_{\infty} & =0 \text { on } \partial \Omega .
\end{array}\right.
$$

Furthermore, $v_{0} \not \equiv 0$ and $0 \leq v_{0} \leq 1$. Thus, $0 \leq v_{0} \leq v_{\infty} \leq 1$ and even $v_{\infty}>0$, $v_{\infty}>v_{0}$ in $\bar{\Omega}$ from the strong elliptic maximum principle.

Take any point $x_{0} \in \mathbb{R}^{N}$ such that $\left|x_{0}\right|=R_{0}+R$. By continuity, there exists $\eta_{0}>1$ such that

$$
v_{\infty}(x)>\varepsilon \psi_{R}\left(x-\eta x_{0}\right) \text { for all } x \in \overline{B\left(\eta x_{0}, R\right)}
$$

and for all $\eta \in\left[1, \eta_{0}\right]$. Notice that $\overline{B\left(\eta x_{0}, R\right)} \subset \bar{\Omega}$ for all $\eta \geq 1$. Say

$\eta^{*}=\sup \left\{\eta \geq 1, v_{\infty}(x)>\varepsilon \psi_{R}\left(x-\eta^{\prime} x_{0}\right)\right.$ for all $x \in \overline{B\left(\eta^{\prime} x_{0}, R\right)}$ and for all $\left.\eta^{\prime} \in[1, \eta]\right\}$.

One has $\eta^{*} \geq \eta_{0}>1$, and one shall prove that $\eta^{*}=+\infty$. Assume on the contrary that $\eta^{*}$ is finite. By continuity,

$$
v_{\infty}(x) \geq \varepsilon \psi_{R}\left(x-\eta^{*} x_{0}\right) \text { for all } x \in \overline{B\left(\eta^{*} x_{0}, R\right)},
$$

and there exists a point $x^{*} \in \overline{B\left(\eta^{*} x_{0}, R\right)}$ such that $v_{\infty}\left(x^{*}\right)=\varepsilon \psi_{R}\left(x^{*}-\eta^{*} x_{0}\right)$. From the strong elliptic maximum principle it follows that

$$
v_{\infty}(x)=\varepsilon \psi_{R}\left(x-\eta^{*} x_{0}\right) \text { for all } x \in \overline{B\left(\eta^{*} x_{0}, R\right)} .
$$

But $v_{\infty}(x)>0=\varepsilon \psi_{R}\left(x-\eta^{*} x_{0}\right)$ for all $x \in \partial B\left(\eta^{*} x_{0}, R\right)(\subset \bar{\Omega})$, and one has then reached a contradiction. Thus, $\eta^{*}=+\infty$ and, since this is true for each $x_{0}$ such that $\left|x_{0}\right|=R_{0}+R$, one infers that

$$
v_{\infty}(x) \geq \varepsilon \psi_{R}(0) \text { for all } x \in \mathbb{R}^{N} \text { such that }|x| \geq R_{0}+R \text {. }
$$

Hence

$$
\inf _{\mathbb{R}^{N} \backslash B_{R_{0}+R}} v_{\infty}>0 .
$$

Since $v_{\infty}$ is continuous and positive in $\bar{\Omega}$, it follows that

$$
m=\inf _{\bar{\Omega}} v_{\infty}>0 .
$$

If $m$ is reached at some point $x \in \bar{\Omega}$, the strong elliptic maximum principle and Hopf lemma yield $m \geq 1$, since $f>0$ in $(0,1)$. Then $v_{\infty} \equiv 1$ (remember that $v_{\infty} \leq 1$ in $\bar{\Omega})$. If $m$ is not attained, there exists a sequence of points $\left(x_{n}\right)_{n \in \mathbb{N}}$ in $\bar{\Omega}$ such that $\left|x_{n}\right| \rightarrow+\infty$ and $v_{\infty}\left(x_{n}\right) \rightarrow m$ as $n \rightarrow+\infty$. The functions $w_{n}(x)=v_{\infty}\left(x+x_{n}\right)$ then converge locally uniformly in $\mathbb{R}^{N}$, up to extraction of some subsequence, to a classical solution $w_{\infty}$ of $\Delta w_{\infty}+f\left(w_{\infty}\right)=0$ in $\mathbb{R}^{N}$ with $m=w_{\infty}(0) \leq w_{\infty} \leq 1$ in $\mathbb{R}^{N}$. One concludes as above that $m=1$.

Therefore, $v_{\infty} \equiv 1$ in $\bar{\Omega}$. Since $u(1+t, x) \geq v(t, x)$ for all $t \geq 1$ and $x \in \bar{\Omega}$, it follows that

$$
\liminf _{t \rightarrow+\infty} \min _{x \in K} u(t, x) \geq 1,
$$

for all compact subset $K \subset \bar{\Omega}$. Together with (3.1), this completes the proof of Lemma 3.1 
Lemma 3.2. Let $u(t, x)$ be a solution of (1.6) with $\Omega=\mathbb{R}^{N}$ and with an initial condition $u_{0} \not \equiv 0$ which is nonnegative, continuous and bounded. Let $g: \mathbb{R}_{+} \rightarrow \mathbb{R}$ be of class $C^{1}$ and such that $g(0)=g(1)=0, g^{\prime}(0)>0, g>0$ on $(0,1)$ and $g<0$ on $(1,+\infty)$. Then, for all $0 \leq c<2 \sqrt{g^{\prime}(0)}$ and for all $e \in \mathbb{R}^{N}$ with $|e|=1$,

$$
u(t, x+c t e) \rightarrow 1
$$

locally uniformly in $x \in \mathbb{R}^{N}$ as $t \rightarrow+\infty$.

This lemma could actually follow from a result by Aronson and Weinberger [1], which was based on the construction of subsolutions involving planar travelling fronts, for the parabolic problem. We present a simpler proof here, which is mainly based on elliptic arguments.

Notice also that the case $c=0$ is included in Lemma 3.1.

Proof of Lemma 3.2, As in Lemma 3.1, one knows that

$$
\limsup _{t \rightarrow+\infty}\left(\sup _{x \in \mathbb{R}^{N}} u(t, x)\right) \leq 1 .
$$

Let $e \in \mathbb{R}^{N}$ be fixed such that $|e|=1$ and let $0 \leq c<2 \sqrt{g^{\prime}(0)}$. Let $R>0$ be large enough so that $\lambda_{R}+c^{2} / 4<g^{\prime}(0)$, where $\left(\lambda_{R}, \psi_{R}\right)$ is the pair of the first eigenvalue and the first eigenfunction of problem (3.2) in the ball $B_{R}$. Since $u$ is continuous and $u(t, x)>0$ for all $t>0$ and $x \in \mathbb{R}^{N}$, one can choose $\varepsilon>0$ small enough so that

$$
\forall x \in \overline{B_{R}}, \quad u(1, x+c e) \geq \varepsilon e^{-c e \cdot x / 2} \psi_{R}(x)=: w_{0}(x) .
$$

Decreasing $\varepsilon>0$ if need be, one can assume that $w_{0} \leq 1$ in $B_{R}$ and

$$
\Delta w_{0}+c e \cdot \nabla w_{0}+g\left(w_{0}\right)=-\left(\lambda_{R}+\frac{c^{2}}{4}\right) w_{0}+g\left(w_{0}\right) \geq 0 \text { in } B_{R} .
$$

Since the function $(t, x) \mapsto v(t, x):=u(t, x+c t e)$ satisfies the equation

$$
v_{t}=\Delta v+c e \cdot \nabla v+g(v)
$$

it then follows that $v(1+t, x) \geq w(t, x)$ for all $t \geq 0$ and $x \in \mathbb{R}^{N}$, where $w$ satisfies the same equation as $v$ with initial condition $w(0, x)=w_{0}(x)$ if $x \in B_{R}$ and $w(0, x)=0$ if $|x| \geq R$.

Furthermore, from the choice of $\varepsilon, w(t, x)$ is nondecreasing in $t$ for all $x \in \mathbb{R}^{N}$ and converges as $t \rightarrow+\infty$ locally uniformly in $x \in \mathbb{R}^{N}$ to a classical solution $w_{\infty}$ of

$$
\Delta w_{\infty}+c e \cdot \nabla w_{\infty}+g\left(w_{\infty}\right)=0 \text { in } \mathbb{R}^{N}
$$

such that $0 \leq w_{\infty} \leq 1$ in $\mathbb{R}^{N}$ and $w_{\infty} \geq w_{0}$ in $B_{R}$. It follows from Proposition 1.14 in [7] that $w_{\infty} \equiv 1$.

Therefore, $\liminf _{t \rightarrow+\infty} \min _{x \in K} u(t, x+c t e) \geq 1$ for all compact subsets $K \subset$ $\mathbb{R}^{N}$. The proof of Lemma 3.2 is thus complete.

Proof of Theorem 1.9. As already underlined, one only has to prove formula (1.11). Let $u$ solve (1.6) with an initial condition $u_{0} \in \mathcal{E}$. Under the assumptions of Theorem 1.9, the exterior domain $\Omega$ satisfies the extension property, whence

$$
\max _{|x| \geq c t, x \in \bar{\Omega}} u(t, x) \rightarrow 0 \text { as } t \rightarrow+\infty,
$$

as soon as $c>2 \sqrt{f^{\prime}(0)}$. 
On the other hand, one easily obtains as usual that

$$
\limsup _{t \rightarrow+\infty} \sup _{x \in \bar{\Omega}} u(t, x) \leq 1
$$

Therefore, one only has to prove that $\liminf _{t \rightarrow+\infty} \min _{|x| \leq c t, x \in \bar{\Omega}} u(t, x) \geq 1$ if $0 \leq c<2 \sqrt{f^{\prime}(0)}$

Let $c$ be fixed such that $0 \leq c<2 \sqrt{f^{\prime}(0)}$ and let $\varepsilon \in(0,1)$ be fixed. It follows from Lemma 3.1 that there exists $t_{0}>0$ such that

$$
\forall t \geq t_{0}, \forall x \in \partial \Omega, \quad u(t, x) \geq 1-\varepsilon .
$$

Now let $g$ be a $C^{1}$ function such that $g \leq f$ in $[0,+\infty), g(0)=g(1-\varepsilon)=0, g>0$ in $(0,1-\varepsilon), g<0$ in $(1-\varepsilon,+\infty)$ and $g^{\prime}(0)=f^{\prime}(0)$. Let $v_{0}$ be a continuous and compactly supported function defined in $\mathbb{R}^{N}$ such that $0 \leq v_{0} \leq 1-\varepsilon$ and $v_{0} \not \equiv 0$. Assume furthermore that $v_{0}$ is radially symmetric, nonincreasing with respect to $r=|x|$ and that $u\left(t_{0}, x\right) \geq v_{0}(x)$ for all $x \in \bar{\Omega}$. Lastly, let $v(t, x)$ be the solution of (1.6) in $\mathbb{R}^{N}$, with nonlinearity $g$ instead of $f$, and initial condition $v_{0}$.

It follows by construction of $g$ that $v(t, x) \leq 1-\varepsilon$ for all $t \geq 0$ and $x \in \mathbb{R}^{N}$. Therefore, $u\left(t+t_{0}, x\right) \geq 1-\varepsilon \geq v(t, x)$ for all $t \geq 0$ and $x \in \partial \Omega$. The above assumptions on $g$ and $v_{0}$ then yield that

$$
\forall t \geq 0, \forall x \in \bar{\Omega}, \quad u\left(t+t_{0}, x\right) \geq v(t, x) .
$$

Thus,

$$
\begin{aligned}
\liminf _{t \rightarrow+\infty} \min _{|x| \leq c t, x \in \bar{\Omega}} u(t, x) & \geq \liminf _{t \rightarrow+\infty} \min _{|x| \leq c t+c t_{0}, x \in \bar{\Omega}} v(t, x) \\
& \geq \liminf _{t \rightarrow+\infty} \min _{|x| \leq c t+c t_{0}, x \in \mathbb{R}^{N}} v(t, x) .
\end{aligned}
$$

On the other hand, $v$ stays radially symmetric in $\mathbb{R}^{N}$ and nonincreasing with respect to $r=|x|$ for all time $t \geq 0$. Therefore,

$$
\liminf _{t \rightarrow+\infty} \min _{|x| \leq c t, x \in \bar{\Omega}} u(t, x) \geq \liminf _{t \rightarrow+\infty} v\left(t, c\left(t+t_{0}\right) e\right)
$$

for any given direction $e \in \mathbb{S}^{N-1}$. However,

$$
\liminf _{t \rightarrow+\infty} v\left(t, c\left(t+t_{0}\right) e\right)=1-\varepsilon
$$

by applying the conclusion of Lemma 3.2 to the function $g$ (remember that $0 \leq c<$ $2 \sqrt{f^{\prime}(0)}=2 \sqrt{g^{\prime}(0)}$ from the choice of $\left.g\right)$.

Since $\varepsilon \in(0,1)$ was arbitrary, one concludes that

$$
\liminf _{t \rightarrow+\infty} \min _{|x| \leq c t, x \in \bar{\Omega}} u(t, x) \geq 1 .
$$

Then, we may conclude that

$$
\lim _{t \rightarrow+\infty} \max _{|x| \leq c t, x \in \bar{\Omega}}|u(t, x)-1|=0
$$

for all $c \in\left[0,2 \sqrt{f^{\prime}(0)}\right)$, and the proof of Theorem 1.9$]$ is complete.

The same type of argument as above gives a lower bound for the spreading speeds $w^{*}\left(e, u_{0}\right)$ and $w^{*}\left(e, z, u_{0}\right)$ in a domain $\Omega$ containing a semi-infinite cylinder in the 
direction $e$, with large enough section. Namely, let us turn to the

Proof of Proposition 1.11. Fix $\varepsilon \in\left(0,2 \sqrt{f^{\prime}(0)}\right]$ and $R_{0}>0$ large enough so that

$$
\forall R \geq R_{0}, \quad \lambda_{R}+\frac{\left(2 \sqrt{f^{\prime}(0)}-\varepsilon\right)^{2}}{4}<f^{\prime}(0),
$$

where $\left(\lambda_{R}, \psi_{R}\right)$ is the pair of the first eigenvalue and the first eigenfunction of problem (3.2) in the ball $B_{R}$. Assume now that $\Omega$ satisfies (1.12) for some $A \in \mathbb{R}$, $x_{0} \in \mathbb{R}^{N}$ and $R>R_{0}$. Fix any $R^{\prime}$ such that $R_{0} \leq R^{\prime}<R$ and set

$$
z_{0}=x_{0}-\left(x_{0} \cdot e\right) e+\left(A+1+R^{\prime}\right) e .
$$

The assumption (1.12) implies that

$$
\forall s \geq 0, \quad \Omega \supset \overline{B\left(z_{0}+s e, R^{\prime}\right)} .
$$

As in the proof of Lemma 3.2 , there exists $\eta>0$ small enough so that

$$
\forall x \in \overline{B_{R^{\prime}}}, \quad u\left(1, x+z_{0}\right) \geq \eta e^{-\left(2 \sqrt{f^{\prime}(0)}-\varepsilon\right) e \cdot x / 2} \psi_{R^{\prime}}(x)=: w_{0}(x)
$$

and $w_{0} \leq 1$ in $\overline{B_{R^{\prime}}}$. From the choice of $R_{0}$, the function $w_{0}$ is a subsolution of

$$
\Delta w_{0}+\left(2 \sqrt{f^{\prime}(0)}-\varepsilon\right) e \cdot \nabla w_{0}+f\left(w_{0}\right) \geq 0 \text { in } B_{R^{\prime}} .
$$

The function $v(t, x)=u\left(t+1, x+z_{0}+\left(2 \sqrt{f^{\prime}(0)}-\varepsilon\right) t e\right)$ satisfies

$$
v_{t}=\Delta v+\left(2 \sqrt{f^{\prime}(0)}-\varepsilon\right) e \cdot \nabla v+f(v)
$$

in particular for all $t \geq 0$ and $x \in \overline{B_{R^{\prime}}}$. Furthermore, $v(t, x) \geq 0$ for all $x \in \partial B_{R^{\prime}}$. It follows from the maximum principle that

$$
v(t, x) \geq w(t, x) \text { for all } t \geq 0 \text { and for all } x \in \overline{B_{R^{\prime}}},
$$

where $w$ solves the same equation as $v$ in $B_{R^{\prime}}$, with initial condition $w(0, x)=w_{0}(x)$ in $B_{R^{\prime}}$ and boundary condition $w(t, x)=0$ for all $t \geq 0$ and $x \in \partial B_{R^{\prime}}$. Furthermore, $0 \leq w(t, x) \leq 1$ for all $t \geq 0$ and $x \in \overline{B_{R^{\prime}}}$, and $w$ is nondecreasing in $t$ for all $x \in \overline{B_{R^{\prime}}}$. Standard parabolic estimates imply that $w(t, x) \rightarrow w_{\infty}(x)$ as $x \rightarrow+\infty$, where $w_{\infty}$ satisfies the corresponding elliptic equation and $w_{\infty}(x) \geq w_{0}(x)$ for all $x \in \overline{B_{R^{\prime}}}$.

As a consequence,

$$
\forall x \in B_{R^{\prime}}, \quad \liminf _{t \rightarrow+\infty} u\left(t+1, x+z_{0}+\left(2 \sqrt{f^{\prime}(0)}-\varepsilon\right) t e\right) \geq w_{\infty}(x)>0 .
$$

Thus, $w^{*}\left(e, z, u_{0}\right) \geq 2 \sqrt{f^{\prime}(0)}-\varepsilon$ for all $u_{0} \in \mathcal{E}$ and $z \in \mathbb{R}^{N}$ such that $\mid z-z_{0}-$ $\left(\left(z-z_{0}\right) \cdot e\right) e \mid<R^{\prime}$. Since this is true for all $R^{\prime} \in\left[R_{0}, R\right)$, one concludes that

$$
w^{*}\left(e, z, u_{0}\right) \geq 2 \sqrt{f^{\prime}(0)}-\varepsilon
$$

for all $u_{0} \in \mathcal{E}$ and $z \in \mathbb{R}^{N}$ such that $\left|z-z_{0}-\left(\left(z-z_{0}\right) \cdot e\right) e\right|<R$.

Together with formula (1.7) of Proposition 1.5, this completes the proof of (1.13).

Remark 3.3. The above arguments imply that if

$$
\Omega \supset\left\{x \in \mathbb{R}^{N}, x \cdot e>A, x \cdot e^{\prime}>B\right\}
$$

for some $(A, B) \in \mathbb{R}^{2}$ and $e^{\prime} \in \mathbb{S}^{N-1}$ with $e^{\prime} \cdot e=0$, then, for all $\varepsilon>0$, there exists $R_{0}>0$ such that $w^{*}\left(e, z, u_{0}\right) \geq 2 \sqrt{f^{\prime}(0)}-\varepsilon$ for all $u_{0} \in \mathcal{E}$ and

$$
z \in \bigcup_{R \geq R_{0}, z_{0} \in \mathbb{R}^{N}, z_{0} \cdot e^{\prime}>B+R} B\left(z_{0}, R\right)
$$


Therefore, $w^{*}\left(e, z, u_{0}\right) \geq 2 \sqrt{f^{\prime}(0)}$ for all $u_{0} \in \mathcal{E}$ and $z$ such that $z \cdot e^{\prime}>B$. This result corresponds to Corollary 1.12

\section{Domains With ZERO OR INFINITE SPREADING SPEEDS, OR SPREADING SPEEDS DEPENDING ON $z$}

This section is devoted to the construction of some particular domains for which the spreading speeds may be zero, infinite, or may depend on the position $z$. All these constructions rely on estimates on the linear heat equation (see formula (2.19) in Proposition 2.5).

\subsection{Domains for which $w^{*}\left(e, z, u_{0}\right)$ depends on $z$.}

End of the proof of Proposition 1.5. Up to translation and rotation, one can assume, say, that $e=(1,0, \ldots, 0)$ and $z=(0,2,0, \ldots, 0)$.

Let $\left(a_{n}\right)_{n \in \mathbb{N}}$ be a sequence of positive real numbers such that

$$
\frac{a_{n}}{n} \rightarrow+\infty \text { as } n \rightarrow+\infty
$$

Let $\Gamma$ be the subset of $\mathbb{R}^{2}$ defined by

$$
\Gamma=\left\{\left(x_{1}, 0\right), x_{1} \geq 0\right\} \cup \bigcup_{n \in \mathbb{N}^{*}}\{n\} \times\left[0, a_{n}\right] .
$$

Let $\tilde{\Omega}$ be any open subset of $\mathbb{R}^{2}$ such that

$$
\Gamma \subset \tilde{\Omega} \subset\left\{x \in \mathbb{R}^{2}, d(x, \Gamma)<\frac{1}{3}\right\}
$$

and such that $\Omega_{2}:=\mathbb{R}^{2} \backslash \overline{\tilde{\Omega}}$ is connected, locally $C^{2}$, and satisfies the extension property defined in Section 1 . Here, $d(y, E)$ denotes the euclidean distance of a point $y \in \mathbb{R}^{m}$ to a subset $E \subset \mathbb{R}^{m}$.

We then set $\Omega=\Omega_{2}$ if $N=2$ (see Figure 1 ) and $\Omega=\Omega_{2} \times \mathbb{R}^{N-2}$ if $N \geq 3$. The open set $\Omega$ is clearly strongly unbounded in the direction $e \underline{4}$ But such a domain does not satisfy the assumptions of Theorem 1.6 (more precisely, $\Omega$ does not satisfy Hypothesis $H_{y, y^{\prime}}$, for any $y$ and $y^{\prime}$ such that $\left.y_{2}>1 / 3>-1 / 3>y_{2}^{\prime}\right)$.

Furthermore,

$$
\forall u_{0} \in \mathcal{E}, \quad w^{*}\left(e, u_{0}\right) \leq 2 \sqrt{f^{\prime}(0)}
$$

from Theorem [1.8. On the other hand, since $\Omega \supset\left\{x \in \mathbb{R}^{N}, x_{2}<-1 / 3\right\}$, Corollary 1.12 implies that $w^{*}\left(e, u_{0}\right) \geq 2 \sqrt{f^{\prime}(0)}$ and $w^{*}\left(e, z^{\prime}, u_{0}\right) \geq 2 \sqrt{f^{\prime}(0)}$ for all $u_{0} \in \mathcal{E}$ and $z^{\prime} \in \mathbb{R}^{N}$ such that $z_{2}^{\prime}<-1 / 3$. Hence,

$$
\begin{aligned}
w^{*}\left(e, u_{0}\right)= & w^{*}\left(e, z^{\prime}, u_{0}\right)=2 \sqrt{f^{\prime}(0)} \\
& \text { for all } u_{0} \in \mathcal{E} \text { and } z^{\prime} \in \mathbb{R}^{N} \text { such that } z_{2}^{\prime}<-1 / 3 .
\end{aligned}
$$

Remember that $z=(0,2,0, \ldots, 0)$. Let $\gamma>0$ be any fixed positive real number and let $u_{0}$ be in $\mathcal{E}$. From the construction of $\Omega$, one has that

$$
\forall s \geq 0, \quad \overline{B(z+s e, 1)} \cap \bar{\Omega} \neq \emptyset .
$$

\footnotetext{
${ }^{4}$ This domain was suggested to us by S. Luckhaus. Its complement, $\mathbb{R}^{N} \backslash \Omega$, has the shape of an infinite comb with larger and larger teeth.
} 


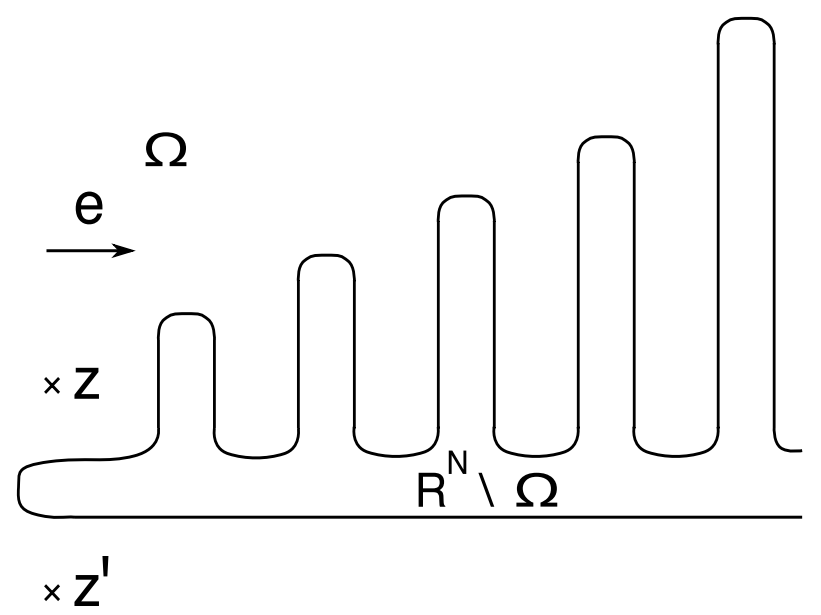

Figure 1. An infinite comb

Let $C_{0}>4$ be given. From Proposition 2.5 and arguing as in the proof of Theorem 1.8 there are some positive constants $C$ and $\delta$ such that

$$
0 \leq u(t, x) \leq e^{f^{\prime}(0) t}\left\|u_{0}\right\|_{L^{\infty}(\Omega)} \int_{\operatorname{supp}\left(u_{0}\right)} C\left(1+(\delta t)^{-N / 2}\right) \exp \left[-\frac{d_{\Omega}(y, x)^{2}}{C_{0} t}\right] d y
$$

for all $t>0$ and $x \in \bar{\Omega}$. Remember that $\operatorname{supp}\left(u_{0}\right)$ denotes the support of $u_{0}$. Since $\operatorname{supp}\left(u_{0}\right)$ is compact, it follows from the construction of $\Omega$ (especially the fact that $a_{n} / n \rightarrow+\infty$ as $\left.n \rightarrow+\infty\right)$ that

$$
y \in \operatorname{supp}\left(u_{0}\right), s \geq \gamma t, x \in \overline{B(z+s e, 1)} \cap \bar{\Omega} \frac{d_{\Omega}(y, x)}{t} \rightarrow+\infty \text { as } t \rightarrow+\infty .
$$

Thus, for all $\beta>0$, there is $t_{0}>0$ such that

$$
0 \leq u(t, x) \leq e^{f^{\prime}(0) t}\left\|u_{0}\right\|_{L^{\infty}(\Omega)} C\left(1+(\delta t)^{-N / 2}\right)\left|\operatorname{supp}\left(u_{0}\right)\right| \times e^{-\beta t}
$$

for all $t \geq t_{0}, s \geq \gamma t$ and $x \in \overline{B(z+s e, 1)} \cap \bar{\Omega}$. Therefore,

$$
\lim _{t \rightarrow+\infty}\left[\sup _{s \geq \gamma t, x \in \overline{B(z+s e, 1)} \cap \bar{\Omega}} u(t, x)\right]=0 .
$$

Since this is true for all $\gamma>0$, one concludes that

$$
w^{*}\left(e, z, u_{0}\right)=0 .
$$

Actually, the same type of argument implies that

$$
w^{*}\left(e, z^{\prime}, u_{0}\right)=0
$$

for all $u_{0} \in \mathcal{E}$ and $z^{\prime} \in \mathbb{R}^{N}$ such that $z_{2}^{\prime}>1 / 2$ (by changing the radius 1 to $1 / 2+\varepsilon$ for some small $\varepsilon=\varepsilon\left(z^{\prime}\right)>0$ ). 


\subsection{Domains with zero spreading speeds.}

Proof of Theorem 1.13, part a). Let us define the curve

$$
\Gamma=\{(t \cos t, t \sin t), t \geq 0\}
$$

and let $\Omega$ be a locally $C^{2}$ open connected subset of $\mathbb{R}^{2}$ satisfying the extension property and such that, say, $\Omega \backslash \overline{B_{2 \pi}}=\{x, d(x, \Gamma)<1\} \backslash \overline{B_{2 \pi}}$. Such a domain $\Omega$ is like a spiral. It is clear that $\Omega$ is strongly unbounded in every unit direction $e$ of $\mathbb{R}^{2}$.

Let $u_{0} \not \equiv 0$ be a nonnegative, continuous and compactly supported function in $\bar{\Omega}$. Let $C_{0}>4, e \in \mathbb{S}^{1}$ be given, and let $R>0$ be such that $\bar{\Omega} \cap \overline{B(s e, R)} \neq \emptyset$ for all $s \geq 0$. From Proposition 2.5 and arguing as in the proof of Theorem 1.8, there are some positive constants $C$ and $\delta$ such that

$$
0 \leq u(t, x) \leq e^{f^{\prime}(0) t}\left\|u_{0}\right\|_{L^{\infty}(\Omega)} \int_{\operatorname{supp}\left(u_{0}\right)} C\left(1+(\delta t)^{-1}\right) \exp \left[-\frac{d_{\Omega}(y, x)^{2}}{C_{0} t}\right] d y
$$

for all $t>0$ and $x \in \bar{\Omega}$. Fix any $\gamma>0$ and $A \geq R$. For all $s \geq 0$ and for all $t>0$, one has

$0 \leq \max _{x \in \overline{B(s e, A)} \cap \bar{\Omega}} u(t, x) \leq C\left\|u_{0}\right\|_{L^{\infty}(\Omega)}\left(1+\delta^{-1} t^{-1}\right) e^{f^{\prime}(0) t} \int_{\operatorname{supp}\left(u_{0}\right)} \exp \left[-\frac{\tilde{r}_{y, s}^{2}}{C_{0} t}\right] d y$

where

$$
\tilde{r}_{y, s}=\frac{\min }{z \in \overline{B(s e, A)} \cap \bar{\Omega}} d_{\Omega}(y, z) .
$$

But, owing to the definition of $\Omega$, there exist $\eta>0$ and $t_{0}>0$ such that

$$
\forall t \geq t_{0}, \forall s \geq \gamma t, \forall y \in \operatorname{supp}\left(u_{0}\right), \tilde{r}_{y, s} \geq \eta t^{2} .
$$

Thus, for all $t \geq t_{0}$,

$$
\begin{aligned}
0 & \leq \sup _{s \geq \gamma t, x \in \overline{B(s e, A)} \cap \bar{\Omega}} u(t, x) \\
& \leq C\left\|u_{0}\right\|_{L^{\infty}(\Omega)}\left(1+\delta^{-1} t^{-1}\right) e^{f^{\prime}(0) t}\left|\operatorname{supp}\left(u_{0}\right)\right| \times e^{-\eta^{2} t^{3} / C_{0}} \rightarrow 0
\end{aligned}
$$

as $t \rightarrow+\infty$.

4.3. Domains with infinite spreading speeds. The proof of part b) of Theorem 1.13 is based on Lemmas 4.1 and 4.2 below. In the remaining part of this section, we fix $N \geq 2$ and we call $\left(x, x^{\prime}\right)$ the coordinates in $\mathbb{R}^{N}$, where $x=x_{1}$ and $x^{\prime}=\left(x_{2}, \ldots, x_{N}\right)$. Let us set $r^{\prime}=\left|x^{\prime}\right|=\sqrt{x_{2}^{2}+\cdots+x_{N}^{2}}$. Let $h: \mathbb{R} \rightarrow \mathbb{R}$ be the function defined for all $s \in \mathbb{R}$ by

$$
h(s)=e^{-e^{s}+s} .
$$

Set

$$
\tilde{\Omega}=\left\{\left(x, x^{\prime}\right) \in \mathbb{R}^{N}, x>A, 0 \leq r^{\prime}<h(x)\right\},
$$

where $A>0$ is a positive real number to be chosen later, and let $\Omega$ be an open connected and locally $C^{2}$ domain such that

$$
\tilde{\Omega} \subset \Omega \subset \tilde{\Omega} \cup\left\{A-1 \leq x \leq A, 0 \leq r^{\prime}<1\right\} .
$$

Such a domain $\Omega$ has the shape of an infinite cusp (see Figure 2), and it obviously does not satisfy the extension property defined in Section 1 


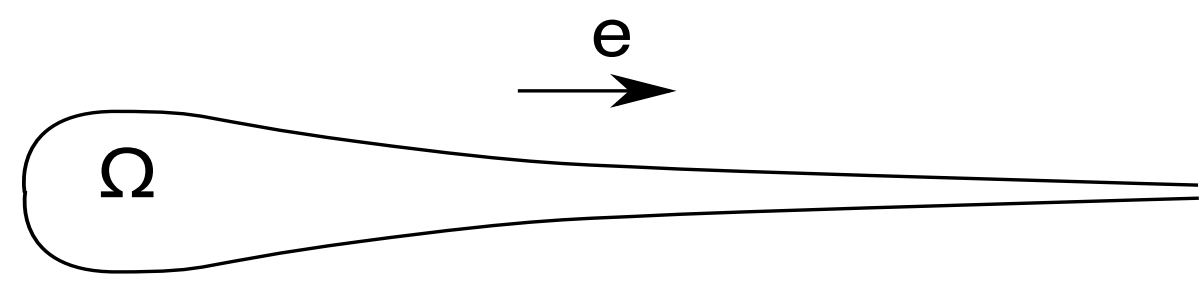

Figure 2. An infinite cusp

Lemma 4.1. Under the above notation, say that

$$
\phi\left(x, x^{\prime}\right)=\phi\left(x, r^{\prime}\right)=\cos r^{\prime}-e^{-x} \cos \left(\sqrt{2} r^{\prime}\right)
$$

for all $\left(x, x^{\prime}\right) \in \mathbb{R}^{N}$. Then there exists $A>0$ large enough such that

$$
\left\{\begin{aligned}
\Delta \phi+\phi & \leq 0 \text { in } \tilde{\Omega} \\
\nu \cdot \nabla \phi & \geq 0 \text { on } \partial \tilde{\Omega} \cap\{x>A\}
\end{aligned}\right.
$$

and $1 / 2 \leq \phi \leq 1$ in $\bar{\Omega}$.

Proof. A straighforward calculation gives us the fact that the function $\phi$ is of class $C^{2}$ in $\mathbb{R}^{N}$ and that

$$
\Delta \phi+\phi=\frac{N-2}{r^{\prime}}\left(-\sin r^{\prime}+\sqrt{2} e^{-x} \sin \left(\sqrt{2} r^{\prime}\right)\right)
$$

if $r^{\prime}>0$. Therefore, $\Delta \phi+\phi \leq 0$ in $\tilde{\Omega}$ for $A$ large enough.

On the other hand, for $\left(x, x^{\prime}\right) \in \partial \tilde{\Omega} \cap\{x>A\}$, one has $r^{\prime}=h(x)$ and

$$
\begin{aligned}
\nu & \cdot \nabla \phi\left(x, x^{\prime}\right) \\
& =\frac{1}{\sqrt{h^{\prime}(x)^{2}+1}}\left(-h^{\prime}(x) e^{-x} \cos (\sqrt{2} h(x))-\sin h(x)+\sqrt{2} e^{-x} \sin (\sqrt{2} h(x))\right) \\
& =\frac{h(x)}{\sqrt{h^{\prime}(x)^{2}+1}}\left(e^{-x}+O\left(h^{2}(x)\right)\right) \\
& \geq 0 \text { for } x \text { large enough. }
\end{aligned}
$$

Lastly, the condition $1 / 2 \leq \phi \leq 1$ in $\bar{\Omega}$ immediately holds if $A$ is large enough. This completes the proof of Lemma 4.1 .

The following lemma provides some lower estimates for the heat kernel in such domains $\Omega$.

Lemma 4.2. Under the assumptions of Lemma 4.1, let $p(t, w, z)$ denote the heat kernel in $\Omega$ with Neumann boundary conditions on $\partial \Omega$. Then there exists a time $T>0$ such that, for all compact subsets $K \subset \bar{\Omega}$,

$$
\inf _{t \geq T, y \in K, z \in \bar{\Omega}} p(t, y, z)>0 .
$$

Proof. Let us first fix $T_{0}>0$ such that

$$
e^{-T_{0}} \leq 1 / 4
$$

Let $K$ be a compact subset of $\bar{\Omega}$. From the strong maximum principle and by continuity there exists $\eta>0$ such that, say,

$$
\forall 1 \leq t \leq 1+T_{0}, \forall y \in K, \forall z=\left(x, x^{\prime}\right) \in \bar{\Omega} \cap\{x \leq A\}, \quad p(t, y, z) \geq \eta .
$$


Let $\eta$ be as above, and let $y$ be any given point in $K$. Let $\varepsilon>0$ and $\beta>0$ be two arbitrary positive real numbers, and let $\bar{u}$ be the function defined for all $t \geq 0$ and $z=\left(x, x^{\prime}\right) \in \bar{\Omega}$ by

$$
\bar{u}(t, z)=p(1+t, y, z)+\varepsilon e^{\beta x} .
$$

One immediately checks that

$$
\bar{u}_{t}-\Delta \bar{u}+\beta^{2} \bar{u}=\beta^{2} p(1+t, y, z)>0
$$

for all $t \geq 0$ and $z \in \Omega$. Furthermore, for all $z=\left(x, x^{\prime}\right) \in \partial \tilde{\Omega} \cap\{x>A\}$ one has

$$
\nu \cdot \nabla \bar{u}=-\frac{\varepsilon \beta e^{\beta x} h^{\prime}(x)}{\sqrt{h^{\prime}(x)^{2}+1}} \geq 0 .
$$

Lastly, $\bar{u}(t, \cdot) \geq \eta$ on $\partial \tilde{\Omega} \cap\{x=A\}$ for all $0 \leq t \leq T_{0}$, because of (4.1).

Now call $\underline{u}$ the function defined for all $t \geq 0$ and $z \in \bar{\Omega}$ by

$$
\underline{u}(t, z)=\eta-2 \eta \phi(z) e^{-\left(1+\beta^{2}\right) t}-\beta^{2} \eta t .
$$

From Lemma 4.1, the function $\underline{u}$ satisfies

$$
\underline{u}_{t}-\Delta \underline{u}+\beta^{2} \underline{u}=2 \eta(\Delta \phi+\phi) e^{-\left(1+\beta^{2}\right) t}-\beta^{4} \eta t \leq 0
$$

for all $z \in \bar{\Omega}$ and $t \geq 0$. Furthermore,

$$
\nu \cdot \nabla \underline{u}=-2 \eta \nu \cdot \nabla \phi e^{-\left(1+\beta^{2}\right) t} \leq 0 \text { on } \partial \tilde{\Omega} \cap\{x>A\}
$$

from Lemma 4.1, and $\underline{u}(t, \cdot) \leq \eta$ in $\bar{\Omega}$ for all $t \geq 0$. Lastly, since $\phi \geq 1 / 2$ in $\bar{\Omega}$, one has that

$$
\bar{u}(0, \cdot) \geq \varepsilon>0 \geq \underline{u}(0, \cdot) \text { in } \bar{\Omega} .
$$

The parabolic maximum principle yields $\bar{u}(t, z) \geq \underline{u}(t, z)$ for all $0 \leq t \leq T_{0}$ and $z \in \overline{\tilde{\Omega}}$. In other words,

$$
\forall 0 \leq t \leq T_{0}, \forall z \in \bar{\Omega}, \quad p(1+t, y, z)+\varepsilon e^{\beta x} \geq \eta-2 \eta \phi(z) e^{-\left(1+\beta^{2}\right) t}-\beta^{2} \eta t .
$$

Since $\varepsilon>0$ and $\beta>0$ were arbitrary, it follows that

$$
\forall 0 \leq t \leq T_{0}, \forall z \in \bar{\Omega}, \quad p(1+t, y, z) \geq \eta-2 \eta \phi(z) e^{-t} .
$$

Since $\phi \leq 1$ in $\bar{\Omega}$, one has $\phi e^{-T_{0}} \leq e^{-T_{0}} \leq 1 / 4$ from the choice of $T_{0}$. Therefore,

$$
\forall z \in \overline{\tilde{\Omega}}, \quad p\left(1+T_{0}, y, z\right) \geq \eta / 2 .
$$

From (4.1), one concludes that $p\left(1+T_{0}, y, z\right) \geq \eta / 2$ for all $z \in \bar{\Omega}$. As a consequence,

$$
p(t, y, z) \geq \eta / 2
$$

for all $t \geq T:=1+T_{0}$ and for all $z \in \bar{\Omega}$. Since $y \in K$ was arbitrary, the proof of Lemma 4.2 is complete (notice that $T$ does not depend on $K$ ).

Let us now turn to the

Proof of Theorem 1.13 , part $b$ ). Let $\Omega$ be as above and such that the conclusion of Lemma 4.1 holds. Let $e=e_{1}=(1,0, \ldots, 0)$. It is clear that $\Omega$ is strongly unbounded in the direction $e$. Let $u_{0} \not \equiv 0$ be a continuous, nonnegative and compactly supported function in $\bar{\Omega}$, and let $u(t, x)$ be the solution of (1.6) with initial condition $u_{0}$. 
Let us first observe that

$$
\forall t \geq 0, \forall x \in \bar{\Omega}, \quad u(t, x) \geq v(t, x),
$$

where $v$ is the solution of (1.6) with initial condition $v_{0}=\min \left(u_{0}, 1\right)$. Since $0 \leq$ $v(t, x) \leq 1$ for all $t \geq 0$ and $x \in \bar{\Omega}$, and since $f \geq 0$ in $[0,1]$, one obtains

$$
\forall t \geq 0, \forall x \in \bar{\Omega}, \quad v(t, x) \geq V(t, x),
$$

where $V$ solves the heat equation $V_{t}=\Delta V$ with Neumann boundary conditions on $\partial \Omega$ and initial condition $v_{0}$.

Therefore, under the notation of Lemma 4.2, one has

$$
\forall t \geq 0, \forall x \in \bar{\Omega}, \quad u(t, x) \geq V(t, x)=\int_{\operatorname{supp}\left(v_{0}\right)} p(t, y, x) v_{0}(y) d y .
$$

Since $\operatorname{supp}\left(v_{0}\right)\left(=\operatorname{supp}\left(u_{0}\right)\right)$ is a compact subset of $\bar{\Omega}$, Lemma 4.2 implies that there exist $T>0$ and $\delta>0$ such that

$$
\forall t \geq T, \forall y \in \operatorname{supp}\left(u_{0}\right), \forall x \in \bar{\Omega}, \quad p(t, y, x) \geq \delta .
$$

Hence,

$$
u(t, x) \geq \varepsilon:=\delta \int_{\operatorname{supp}\left(u_{0}\right)} v_{0}(y) d y>0
$$

for all $t \geq T$ and $x \in \bar{\Omega}$.

As a consequence, $u(t+T, x) \geq \zeta(t)>0$ for all $t \geq 0$ and $x \in \bar{\Omega}$, where $\zeta$ solves $\dot{\zeta}=f(\zeta)$ with $\zeta(0)=\varepsilon>0$. Since $\zeta(t) \rightarrow 1$ as $t \rightarrow+\infty$ (because of the profile of $f)$, one obtains

$$
\liminf _{t \rightarrow+\infty} \inf _{x \in \bar{\Omega}} u(t, x) \geq 1 .
$$

On the other hand, $u(t, x) \leq \xi(t)$ for all $t \geq 0$ and $x \in \bar{\Omega}$, where $\xi$ solves $\dot{\xi}=f(\xi)$ and $\xi(0)=\max _{\bar{\Omega}} u_{0} \in(0,+\infty)$. Since $\xi(t) \rightarrow 1$ as $t \rightarrow+\infty$, one obtains as usual that

$$
\limsup _{t \rightarrow+\infty} \sup _{x \in \bar{\Omega}} u(t, x) \leq 1 \text {. }
$$

As a conclusion, $u(t, x) \rightarrow 1$ as $t \rightarrow+\infty$ uniformly with respect to $x \in \bar{\Omega}$.

Owing to Definitions 1.2 and 1.3 , it follows that $w^{*}\left(e, z, u_{0}\right)=w^{*}\left(e, u_{0}\right)=+\infty$ for all $z \in \mathbb{R}^{N}$ and $u_{0} \in \mathcal{E}$. This completes the proof of Theorem 1.13 , part b).

\section{ACKNOWLEDGEMENTS}

The authors are thankful to the referees for a careful reading and to Stephan Luckhaus for the conversations about this work and in particular for his suggestion of the example given in the proof of the second part of Proposition 1.5

\section{REFERENCES}

[1] D.G. Aronson, H.F. Weinberger, Multidimensional nonlinear diffusions arising in population genetics, Adv. Math. 30 (1978), pp. 33-76. MR511740 (80a:35013)

[2] B. Audoly, H. Berestycki, Y. Pomeau, Réaction-diffusion en écoulement stationnaire rapide, C. R. Acad. Sci. Paris 328 II (2000), pp. 255-262.

[3] H. Berestycki, The influence of advection on the propagation of fronts in reaction-diffusion equations, In: Nonlinear PDE's in Condensed Matter and Reactive Flows, H. Berestycki and Y. Pomeau, eds., Kluwer Academic Publ., 2002, pp. 1-45.

[4] H. Berestycki, F. Hamel, Front propagation in periodic excitable media, Comm. Pure Appl. Math. 55 (2002), pp. 949-1032. MR.1900178 (2003d:35139) 
[5] H. Berestycki, F. Hamel, Generalized travelling waves for reaction-diffusion equations, In: Perspectives in Nonlinear Partial Differential Equations. In honor of Haïm Brezis, Amer. Math. Soc., Contemp. Math. 446, 2007, pp. 101-123. MR2373726

[6] H. Berestycki, F. Hamel, N. Nadirashvili, The principal eigenvalue of elliptic operators with large drift and applications to nonlinear propagation phenomena, Comm. Math. Phys. 253 (2005), pp. 451-480. MR2140256 (2006b:35057)

[7] H. Berestycki, F. Hamel, N. Nadirashvili, The speed of propagation for KPP type problems. I Periodic framework, J. Europ. Math. Soc. 7 (2005), pp. 173-213. MR2127993 (2005k:35186)

[8] H. Berestycki, L. Nirenberg, Travelling fronts in cylinders, Ann. Inst. H. Poincaré, Anal. Non Lin. 9 (1992), pp. 497-572. MR1191008 (93k:35019)

[9] P. Constantin, A. Kiselev, A. Oberman, L. Ryzhik, Bulk burning rate in passive-reactive diffusion, Arch. Ration. Mech. Anal. 154 (2000), pp. 53-91. MR1778121 (2001g:35119)

[10] E.B. Davies, Heat kernels and spectral theory, Cambridge Univ. Press, 1989. MR990239 (90e:35123)

[11] P.C. Fife, J.B. McLeod, The approach of solutions of non-linear diffusion equations to traveling front solutions, Arch. Ration. Mech. Anal. 65 (1977), pp. 335-361. MR0442480 (56:862)

[12] R.A. Fisher, The advance of advantageous genes, Ann. Eugenics 7 (1937), pp. 335-369.

[13] M. Freidlin, On wave front propagation in periodic media, In: Stochastic analysis and applications, ed. M. Pinsky, Advances in Probability and Related Topics 7, Dekker, New York, 1984, pp. 147-166. MR776979 (87d:35065)

[14] M. Freidlin, J. Gärtner, On the propagation of concentration waves in periodic and random media, Sov. Math. Dokl. 20 (1979), pp. 1282-1286. MR.553200 (81d:80005)

[15] T. Gallay, Local stability of critical fronts in nonlinear parabolic pde's, Nonlinearity 7 (1994), pp. 741-764. MR.1275528 (95c:35122)

[16] A. Grigor'yan, Gaussian upper bounds for the heat kernel on arbitrary manifolds, J. Diff. Geom. 45 (1997), pp. 33-52. MR1443330 (98g:58167)

[17] M. Gruber, Harnack inequalities for solutions of general second order parabolic equations and estimates of their Hölder constants, Math. Z. 185 (1984), pp. 23-43. MR724044 (86b:35089)

[18] K.P. Hadeler, F. Rothe, Travelling fronts in nonlinear diffusion equations, J. Math. Biol. 2 (1975), pp. 251-263. MR0411693 (53:15423)

[19] F. Hamel, Formules min-max pour les vitesses d'ondes progressives multidimensionnelles, Ann. Fac. Sci. Toulouse 8 (1999), pp. 259-280. MR1751443 (2001e:35088)

[20] F. Hamel, Qualitative properties of monostable pulsating fronts: exponential decay and monotonicity, J. Math. Pures Appl. 89 (2008), pp. 355-399. MR.2401143

[21] F. Hamel, L. Roques, Uniqueness and stability properties of monostable pulsating fronts, preprint.

[22] S. Heinze, Diffusion-advection in cellular flows with large Peclet numbers, Arch. Ration. Mech. Anal. 168 (2003), pp. 329-342. MR.1994746 (2004e:76037)

[23] S. Heinze, Large convection limits for KPP fronts, preprint.

[24] S. Heinze, G. Papanicolaou, A. Stevens, Variational principles for propagation speeds in inhomogeneous media, SIAM J. Appl. Math. 62 (2001), pp. 129-148. MR.1857539 (2002j:35169)

[25] W. Hudson, B. Zinner, Existence of travelling waves for reaction-diffusion equations of Fisher type in periodic media, In: Boundary Problems for Functional Differential Equations, World Scientific, 1995, pp. 187-199. MR.1375475 (97a:35112)

[26] C.K.R.T. Jones, Spherically symmetric solutions of a reaction-diffusion equation, J. Diff. Eqs. 49 (1983), pp. 142-169. MR704268 (84h:35084)

[27] Ya.I. Kanel', On the stability of solutions of the equations of combustion theory for finite initial functions, Mat. Sbornik 65 (1964), pp. 398-413. MR0177209 (31:1473)

[28] A. Kiselev, L. Ryzhik, Enhancement of the traveling front speeds in reaction-diffusion equations with advection, Ann. Inst. H. Poincaré, Anal. Non Lin. 18 (2001), pp. 309-358. MR.1831659 (2002c:35155)

[29] A.N. Kolmogorov, I.G. Petrovsky, N.S. Piskunov, Étude de l'équation de la diffusion avec croissance de la quantité de matière et son application à un problème biologique, Bulletin Université d'Etat à Moscou, Sér. Internationale A 1 (1937), pp. 1-26.

[30] N.V. Krylov, M.V. Safonov, A property of the solutions of parabolic equations with measurable coefficients, Izv. Akad. Nauk SSSR Ser. Mat. 44 (1980), pp. 161-175. MR563790(83c:35059)

[31] G.M. Lieberman, Second Order Parabolic Differential Equations, World Scientific Publishing Co. Inc., River Edge, NJ, 1996. MR1465184 (98k:35003) 
[32] R. Lui, Biological growth and spread modeled by systems of recursions. I. Mathematical theory, Math. Bios. 93 (1989), pp. 269-295. MR984281 (90g:92069)

[33] A.J. Majda, P.E. Souganidis, Large scale front dynamics for turbulent reaction-diffusion equations with separated velocity scales, Nonlinearity 7 (1994), pp. 1-30. MR1260130 (95e:35180)

[34] J.-F. Mallordy, J.-M. Roquejoffre, A parabolic equation of the KPP type in higher dimensions, SIAM J. Math. Anal. 26 (1995), pp. 1-20. MR1311879 (96a:35090)

[35] H. Matano, Oral communication.

[36] J.-M. Roquejoffre, Eventual monotonicity and convergence to travelling fronts for the solutions of parabolic equations in cylinders, Ann. Inst. H. Poincaré, Anal. Non Lin. 14 (1997), pp. 499-552. MR1464532 (98h:35107)

[37] L. Ryzhik, A. Zlatos, KPP pulsating front speed-up by flows, Commun. Math. Sci. 5 (2007), pp. 575-593. MR2352332(2008h:35200)

[38] N. Shigesada, K. Kawasaki, Biological invasions: theory and practice, Oxford Series in Ecology and Evolution, Oxford: Oxford UP, 1997.

[39] A.I. Volpert, V.A. Volpert, V.A. Volpert, Traveling wave solutions of parabolic systems, Translations of Math. Monographs 140, Amer. Math. Soc., 1994. MR.1297766 (96c:35092)

[40] H.F. Weinberger, On spreading speeds and traveling waves for growth and migration in periodic habitat, J. Math. Biol. 45 (2002), pp. 511-548. MR.1943224 (2004b:92043a)

[41] X. Xin, Existence of planar flame fronts in convective-diffusive periodic media, Arch. Ration. Mech. Anal. 121 (1992), pp. 205-233. MR:1188981 (93m:35110)

[42] A. Zlatos, Sharp asymptotics for KPP pulsating front speed-up and diffusion enhancement by flows, preprint.

EHESS, Centre d'Analyse et Mathématique Sociales, 54 Boulevard Raspail, F-75006 PARIS, France

Université AiX-Marseille III, Laboratoire D'Analyse, Topologie, Probabilités, FaCulté des Sciences et Techniques, Avenue Escadrille Normandie-Niemen, F-13397 MarSeille Cedex 20, France

CNRS, laboratoire d'Analyse, Topologie, Probabilités, CMi, 39 rue F. Joliot-Curie, F-13453 Marseille Cedex 13, France 